\title{
Plasmonic- and dielectric-based structural coloring: from fundamentals to practical applications
}

\author{
Taejun Lee ${ }^{1 \dagger}$, Jaehyuck Jang ${ }^{2 \dagger}$, Heonyeong Jeong ${ }^{1}$ and Junsuk Rho ${ }^{1,2^{*}}$ (D)
}

\begin{abstract}
Structural coloring is production of color by surfaces that have microstructure fine enough to interfere with visible light; this phenomenon provides a novel paradigm for color printing. Plasmonic color is an emergent property of the interaction between light and metallic surfaces. This phenomenon can surpass the diffraction limit and achieve near unlimited lifetime. We categorize plasmonic color filters according to their designs (hole, rod, metal-insulator-metal, grating), and also describe structures supported by Mie resonance. We discuss the principles, and the merits and demerits of each color filter. We also discuss a new concept of color filters with tunability and reconfigurability, which enable printing of structural color to yield dynamic coloring at will. Approaches for dynamic coloring are classified as liquid crystal, chemical transition and mechanical deformation. At the end of review, we highlight a scale-up of fabrication methods, including nanoimprinting, self-assembly and laser-induced process that may enable real-world application of structural coloring.
\end{abstract}

Keywords: Structural color printing, Color filters, Plasmonic color filters, Plasmonics, Dielectric color filters, Metasurfaces, Metamaterials, Sub-wavelength, Nanophotonics, Tunable color filters, Large scale color filters, Up-scale color filters

\section{Introduction}

Color production mechanism are mainly classified by two types: pigmentary or structural coloring. Structural colors, in particular, are caused by microscopic structures that are tiny enough to interfere with visible light. In nature, structural coloring occurs among birds and insects [1-6]. This method of generating colors has inspired the field of structural color printing. Many artificial and biomimetic colors from nature have been reproduced $[7,8]$ and applied to photonic crystal research [9-11]. However, the diffraction limit of light presents a challenge to further development of structural color printing. Recent developments of techniques to fabricate

\footnotetext{
*Correspondence: jsrho@postech.ac.kr

TTaejun Lee and Jaehyuck Jang equally contributed first authorship and equally contributed to this work

${ }^{1}$ Department of Mechanical Engineering, Pohang University of Science and Technology (POSTECH), Pohang 37673, Republic of Korea

Full list of author information is available at the end of the article
}

metal-based structures have shown a way to overcome the diffraction and to approach nano-size resolution.

Surface plasmon resonance (SPR) by the electric field along a metallic surface can confine optical excitation to far below the diffraction limit $[12,13]$. Furthermore, SPRs can be used to manipulate the polarization, phase and intensity of light $[14-18,19,20,21]$. These characteristics offer a capability to generate structural colors from SPRs on plasmonic structures. Plasmonic color filters (PCFs) can have sub-wavelength unit cells and surpass the diffraction limit of light due to SPR from plasmonic structures [22, 23, 24]. Hole-array PCFs achieve high transmittance by exploiting extraordinary optical transmission (EOT) caused by SPRs [15, 25-27].

The metallic layer in PCFs absorbs visible light; this phenomenon can reduce their efficiency. Use of dielectric-based color filters (DCFs) supported by Mie resonance has been suggested as a method to circumvent this problem [28-30]. DCFs have relatively low loss, so they 
can control bandwidth adaptively. Additionally, owing to optically-generated electric and magnetic resonances and low cost, DCFs have substantial potential to complement or even replace pigments and PCFs [31].

Structural coloring has limitations such as static color, limited-scale fabrication and low throughput. Structural filters produce colors that stay mostly static, so the search for a method to tune them is currently an important research topic. Structural color filters that can be tuned by adjusting external factors can manipulate colors by controlling factors such as polarization angle of incident light, alignment of liquid crystals (LCs) [32-35], mechanical strain [36-39] and chemical state [40-43]. In this review, we summarize research on plasmonic color filtering, with a brief explanation of its working principle. We also introduce recent developments in tunable color filtering and large-scale color filtering, which may lead to real-world application.

\section{Plasmonic color filters}

Color filters selectively reflect or transmit light of a target wavelength. A unit (pixel) in an array transmits dominantly one color (e.g., red, green, or blue). Each pixel delivers different color information, and a combination of colored pixels can produce a specific image. A structural color filter has a nano-scale structure that interacts with incident light to reflect or transmit light of a specific wavelength. PCFs that exploit plasmonic resonance are promising candidates to replace conventional pigmentor dye-based color filters. We will review research on PCFs sorted by structure.

\subsection{Nanohole}

Transmission of light through a subwavelength aperture in regularly patterned opaque metal film is enhanced at resonant wavelengths. This phenomenon is called EOT, and is one of the most important recent discoveries in optics [15]. The effect is associated with a coupling between excitation of surface plasmon (SP) and incident light in a metallic surface [44, 45]. The interaction can be manipulated by tuning geometric parameters such as periodicity, size and shape of apertures. This observation has triggered a wide variety of related research [46-51].

In PCFs with asymmetric cross-shaped apertures, one nanoaperture can generate two colors (Fig. 1a). PCFs in aluminium (Al) film can have polarization-adjustable properties that result in selectivity between two transmitted colors [52]. By manipulating geometric parameters such as long arm (LA), short arm (SA) and periodicity of nanocavity apertures, various colors are obtained. Exchanging the polarization directions of the LA and SA result in dual color variations. No color changes in SA-variable palettes appear at a same period when light is transmitted through the LA and polarized along the SA. In contrast, the color changes gradually when light is transmitted through the SA and polarized along the LA (Fig. 1b). Therefore, by controlling polarization direction of incident light an operator can selectively obtain one of two colors from the plasmonic device.

Nanohole-shaped PCFs based on silver (Ag) instead of $\mathrm{Al}$ or gold $\mathrm{Au}$ ) can produce color-enhanced transmissive structural colors [53]. The Ag color diagram has a wide distribution of displayed colors, whereas the $\mathrm{Al}$ and $\mathrm{Au}$ diagrams focus on certain colors (Fig. 1d). Authors also present three pixels which are resists that were fully, partially and barely exposed to electron beam lithography (EBL) (Fig. 1f, red, yellow and white circles). Although the holes are barely exposed, they show no variation of either color or shape compared to fully-exposed pixels. The fabrication method is a simple two-step process of nanoimprinting and depositing the metal (Fig. 1e). Because this fabrication is simple and compatible with large-scale fabrication, commercialization of these devices as color filters is expected.

\subsection{Metal-insulator-metal}

Metal-insulator-metal (MIM) nanoresonators with an insulator sandwiched between two metallic materials can also act as color filters. The key principle of MIM is Fabry-Pérot interferometry, in which interference of light within a resonator selectively filters out light of a certain wavelength. The design of the MIM has evolved from films to 2D metasurfaces with unit structures such as gratings or posts [54-64]. To improve efficiencies or color purity, researchers have changed basic materials [65-67].

A research based on Ag-alumina $\left(\mathrm{Al}_{2} \mathrm{O}_{3}\right)-\mathrm{Ag}$ exploiting tandem nanodisks demonstrates a wide range of color generation by adjusting geometric parameters such as periodicity and radius of structures (Fig. 2a) [68]. Previous works on plasmonic color printing have mostly concentrated on hue instead of color brightness and saturation. These methods suffer from relatively broad full width at half-maximum, shallow peaks, and dips in spectra. A device based on $\mathrm{Ag}-\mathrm{Al}_{2} \mathrm{O}_{3}-\mathrm{Ag}$ consists of an array of tandem nanodisks to solve these limitations, and achieved bright and saturated colors with red, green and blue in reflective mode, and cyan, magenta and yellow in transmissive mode (Fig. 2ai, ii). These complementary near-full colors are attributed to a combination of Wood's anomaly and an in-phase electric dipole. This device is relatively easy to fabricate because it uses comparatively large nanodisks.

Plasmonic stereoscopic printing has been demonstrated [69]. The device can produce a great variety of colors in reflective mode by using polarization-dependent 
a
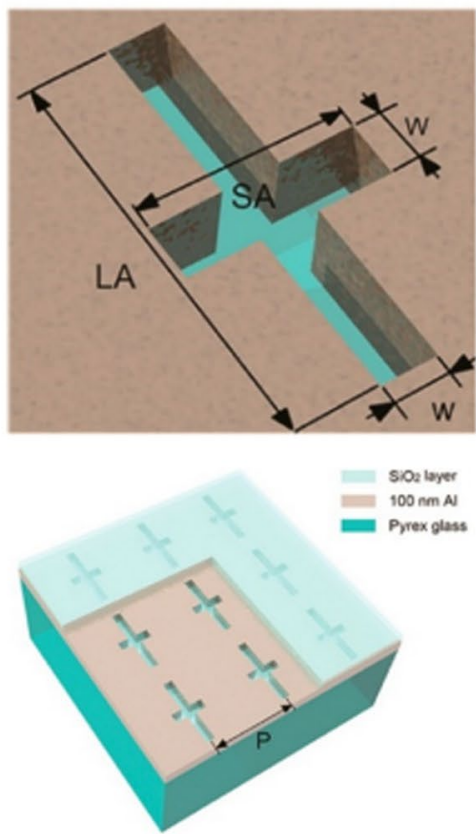

b

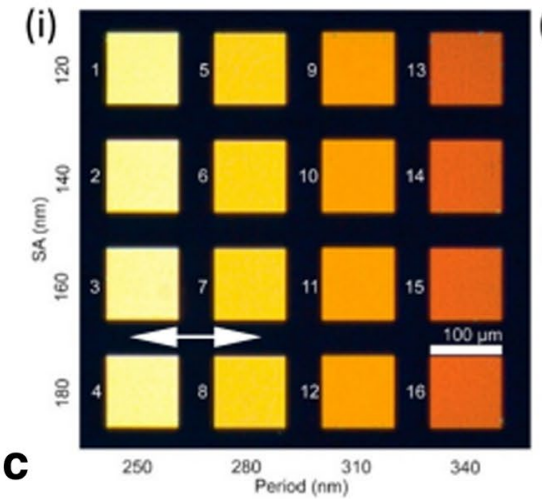

(i)

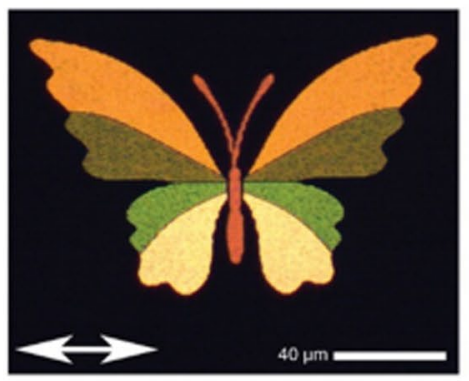

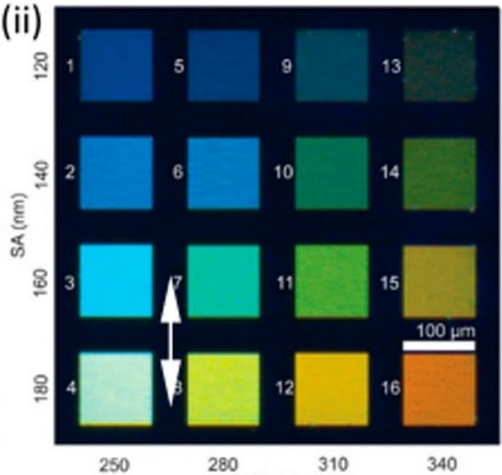

(ii)

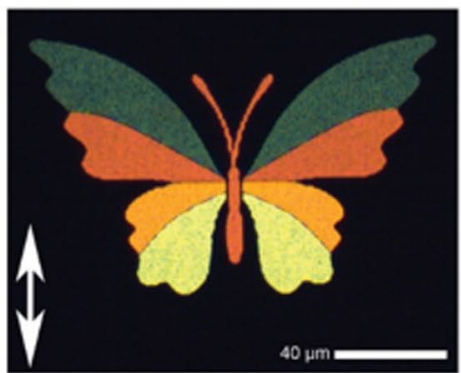

d
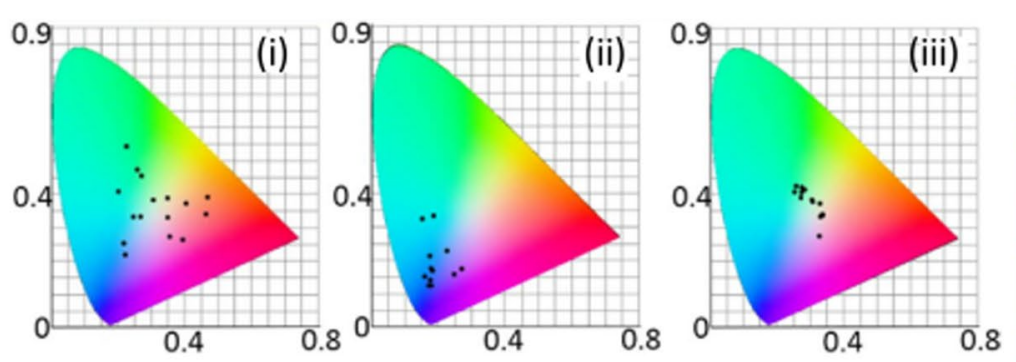

e
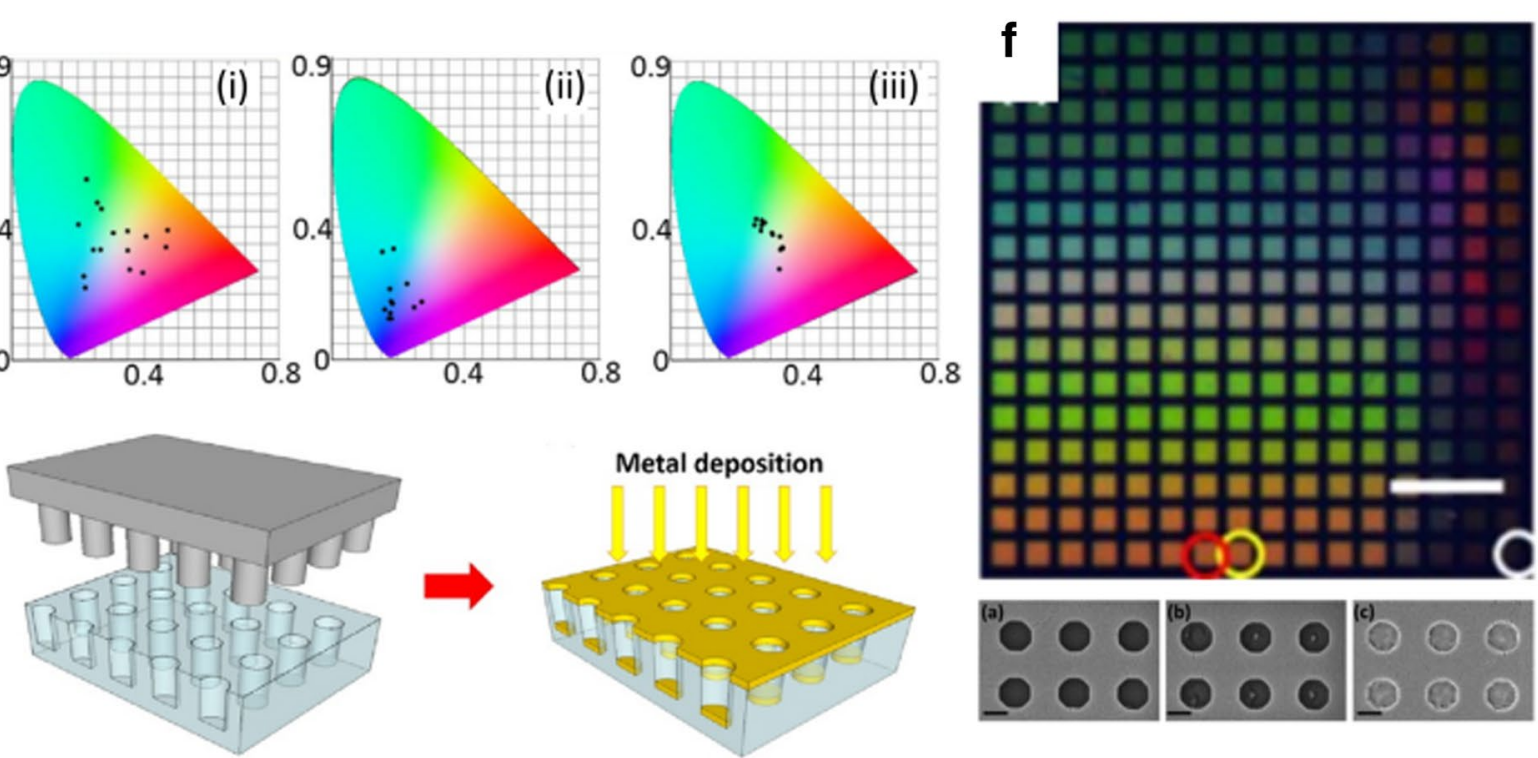

Fig. 1 a Schematics of cross-shaped PCFs (SA short arm, LA long arm. The average dimensions of aperture are $W=30 \pm 2 \mathrm{~nm}, P=340 \mathrm{~nm}$, $S A=120 \pm 5 \mathrm{~nm}$ and $L A=203 \pm 3 \mathrm{~nm}$. b Color palette with variation in polarization. (i) Colors transmitted through the LA resulting from polarization along the SA. (ii) Colors transmitted through the SA resulting from polarization along the LA. c Micrographs of butterfly with wing color variations, when polarized along SA (i) and LA (ii) [52]. d Color distributions simulated in CIE 1931 chromaticity diagram with (i) Ag (ii) Al and (iii) Au. e Two-step fabrication composed of nanoimprint and metal deposition. $\mathbf{f}$ (top) Transmissive microscopic color palette using Ag. (bottom) Three scanning electron microscope (SEM) images of nanohole pixels with 500-nm periodicity and exposure to e-beam dose of 900 (left), 720 (middle) and 500 (right) $\mu \mathrm{C} / \mathrm{cm}^{2}$, corresponding to red, yellow and white circles of top image [53] 


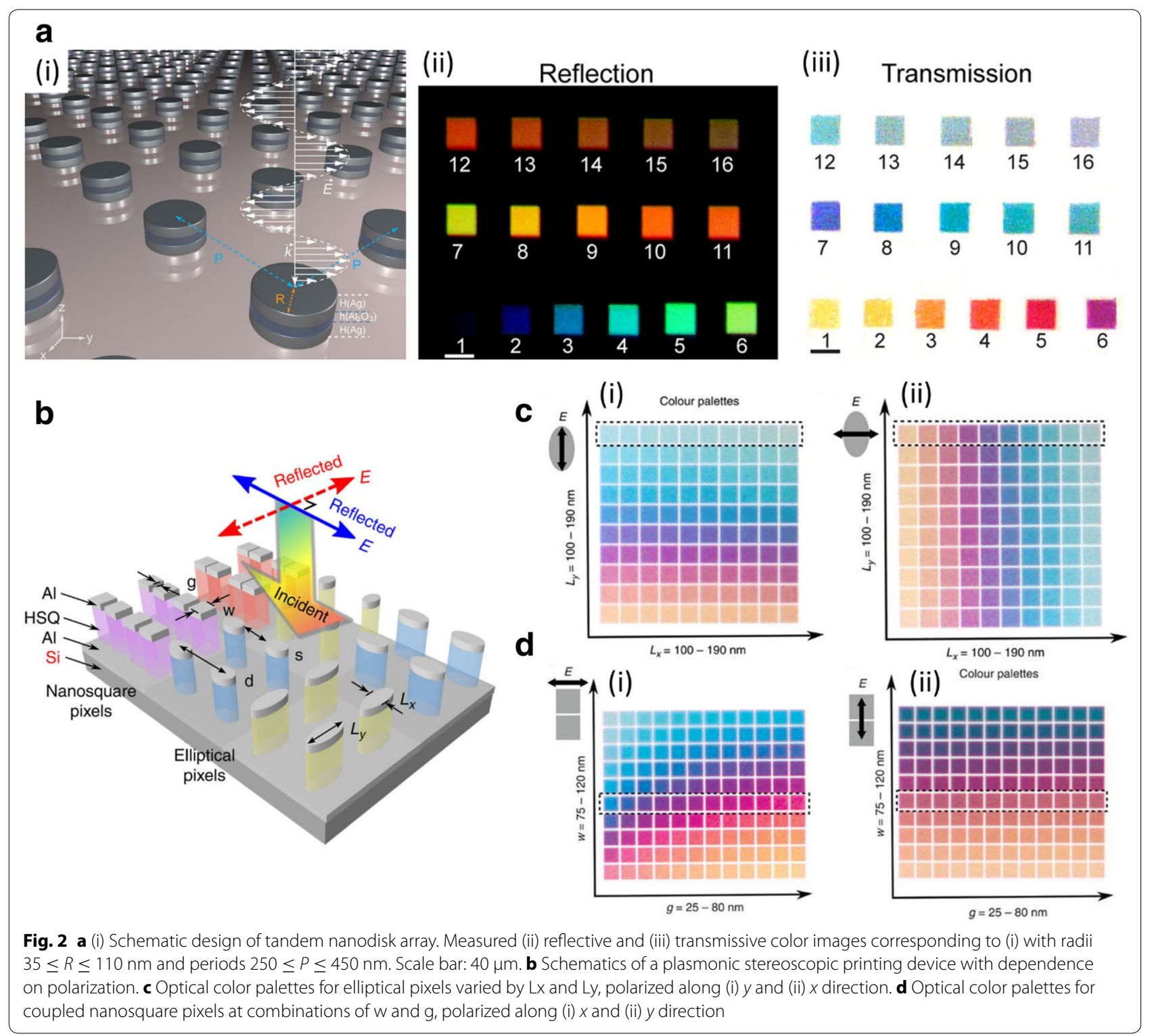

tunable color pixels in pairs of arrays of squares and of ellipses (Fig. 2b). Dual color information can be encoded by two polarization directions in the same pixel (Fig. 2c, d), so this printing technology may have applications as high-density optical data storage, high-resolution 3D display, holograms and anti-counterfeiting measures.

\subsection{Nanorod}

Color imaging devices based on nanorod arrays can be made to demonstrate a color spectrum by manipulating their resonances [70-76]. The generated colors are mainly attributed to an interaction between plasmon resonance that is related to peaks in spectra, and Fano resonance that is related to dips in them. Although the influence of the Fano resonance is not critical, it helps to narrow the spectral peaks to sharpen and enrich the colors. Near-full-color printing has been achieved using $\mathrm{Ag}$ and $\mathrm{Au}$ nanodisks on a backreflector that encodes the color information (Fig. 3a) [77]. Each color pixel consists of four nanodisks; the results are achieved by varying the diameter of disks and the gap between them, but are not affected by their periodicity. Use of a metallic capping layer can also intensify the color (Fig. 3b). This approach is compatible with nanoimprint lithography, and may therefore be amenable to scale-up and high-throughput fabrication.

A novel nanorod-shaped plasmonic pixel can produce optical resonances over the entire visible spectrum 


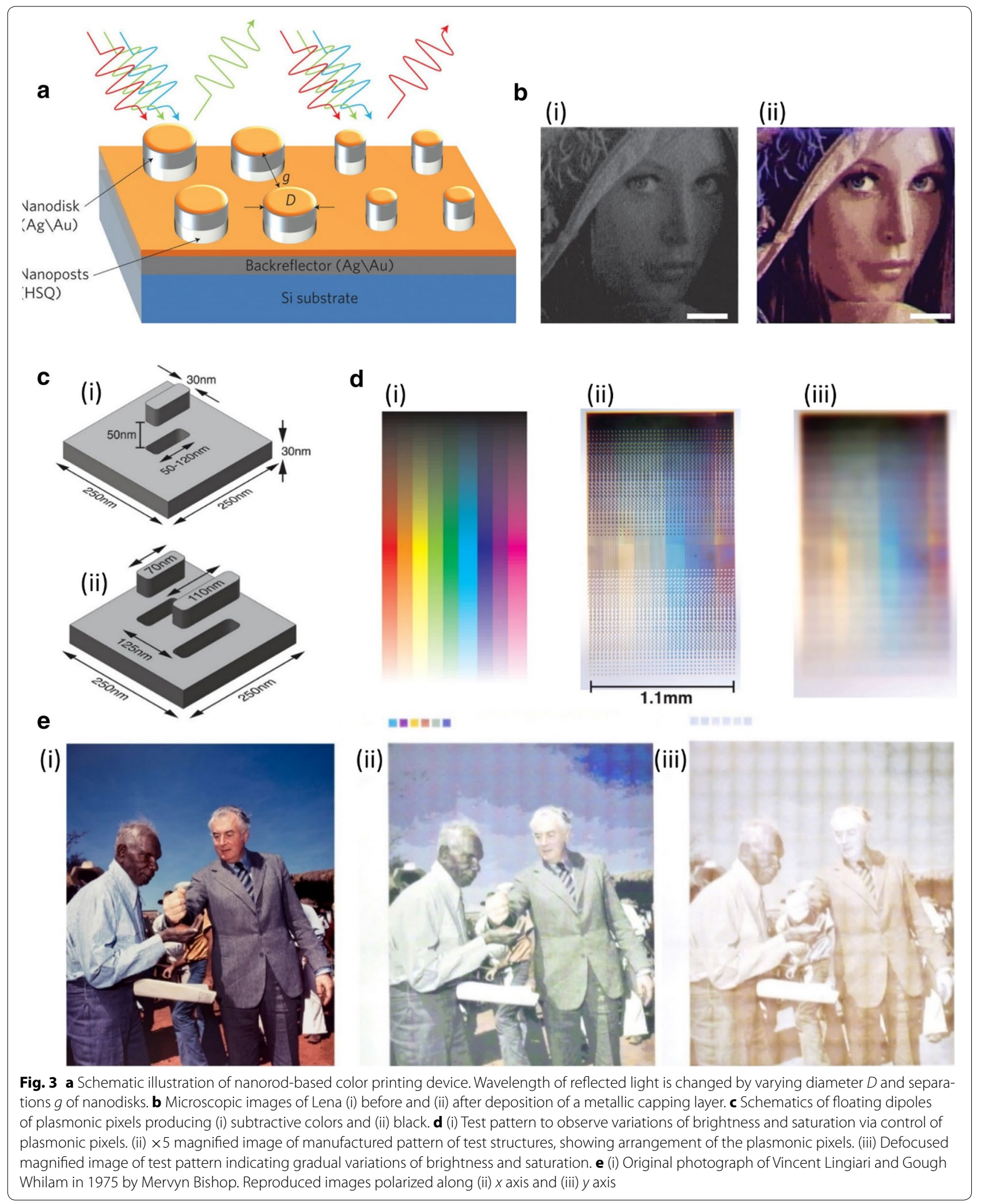


[78]. The floating plasmonic pixel acts as a plasmonic nearly-perfect absorber with narrow bandwidth, and thereby produces immensely-saturated subtractive colors (Fig. 3ci). The yellow, magenta and cyan of subtractive colors are generated at dipole lengths of 70, 90 and $120 \mathrm{~nm}$, respectively. Experimental results agree well with simulation, except in saturation of the yellow. The discrepancy may result from fabrication imprecision, which broadens a shape of the resonance, but can be overcome by manipulating evaporation parameters. Black is generated by a two-connected floating dipole that acts as a near-perfect absorber, so broad absorption appears in the visible spectrum (Fig. 3c) [30]. This approach can also adjust the color response by tuning geometric parameters such as nanoantenna length and gap between antenna and film.

\subsection{Grating}

Development of grating-based 1D PCFs has achieved $>70 \%$ average efficiencies of either transmission or reflection $[49,79-89]$. Due to structural periodicity, these devices usually exhibit angle-sensitivity and the momentum-matching constraint of surface plasmon polaritons (SPPs). These problems have been resolved simultaneously using randomly-arrayed nanostructures [90].

Polarization-dependence can also appear in gratingbased color-printing devices. A silver-based grating color filter that uses a plasmonic phase retarder causes dependence on incident polarization by simple rotation of a polarizer [91]. By changing the different polarizer settings, the same input image can be given very different color properties (Fig. 4a). The $45^{\circ}$ and $135^{\circ}$ polarization angles generate strongly-contrasting colors of purple, dark blue, orange and yellow. Photomask used to make the images is divided into five areas according to the grating thickness of the Ag layer. The analyzing polarizer has two inverse regions to improve optical appearance. Polarization-sensitive color printing technology has potential applications in security [92, 93], sensing [94], polarizing detectors [76] and magneto-optics [95].

A combined design of MIM and grating can efficiently separate white light into a certain wavelength with distinct color (Fig. 4b) [62]. The MIM-grating color filter consists of $\mathrm{Al}-\mathrm{ZnSe}-\mathrm{Al}$ resonators. Diffraction at the bottom $\mathrm{Al}$ grating layer helps couple incident light to plasmon waveguide modes; scattering at the top $\mathrm{Al}$ grating layer reconverts the detained plasmon to a propagating wave. The $\mathrm{ZnSe}$ layer with $100-\mathrm{nm}$ thickness encourages a coupling of SP in top and bottom, so that the nanoresonators effectively actualize a conversion of photon-plasmon-photon at the resonant wavelengths. This MIM grating design has many advantages of compactness, effective transmission and narrow passband. The dependence on polarization eliminates the need for an individual polarizer layer, so these MIM/grating devices may have applications in liquid crystal displays [96].

\section{All-dielectric structure supported by Mie resonance}

Devices that use metallic nanostructures to induce structural colors can manipulate light absorption and scattering beyond the optical diffraction limit. These devices have advantages of compactness, high resolution, robustness, and compatibility with integration in various devices. DCFs have been studied to seek capabilities that complement limitations of metal, such as high loss that leads to peak broadening. Such devices exploit Mie resonance based on Mie scattering that depends on both the geometry and size of particles. In principle, dielectric nanoparticles (NPs) with high refractive index $n$ can affect the results, with both the electric dipole and magnetic dipole having comparable contributions, whereas PCFs mainly control the electric dipole. The resonant magnetic response is caused by coupling of incident light toward the circular displacement current of the electric field as a result of retardation of phase and field penetration. This series of processes arises when the wavelength $\lambda$ of the light is approximately the diameter $2 R$ of inner particles $(2 R \approx \lambda / n)$. This mechanism may also provide an opportunity to design DCFs that use Mie resonances to exploit higher-order multipoles [31].

Optically-generated resonant magnetic responses in dielectric NPs have been observed in particles with different geometries such as ring [97], spheroid [98, 99], disk and cylinder [100] and sphere [101]. This diversity of optically-active shapes provides an opportunity to create diverse all-dielectric nanostructures by varying the geometric parameters of NPs to adjust both magnetic and electric resonances. Before choosing design of structure, its material of structure must be selected, because it effects the optical characteristics of the device such as transmission, reflection, optical loss and efficiency.

Many types of dielectric materials are complementarily available; each has advantages and disadvantages. Several materials cannot be used alone because of high optical losses. Hence, these materials are sometimes used in chemical or mechanical mixtures in which each has a property that compensates for the weakness of another. So far, silicon $(\mathrm{Si})$ has been used most commonly, but many researchers have attempted to find optimal materials suitable for devices that require particular optical properties. 

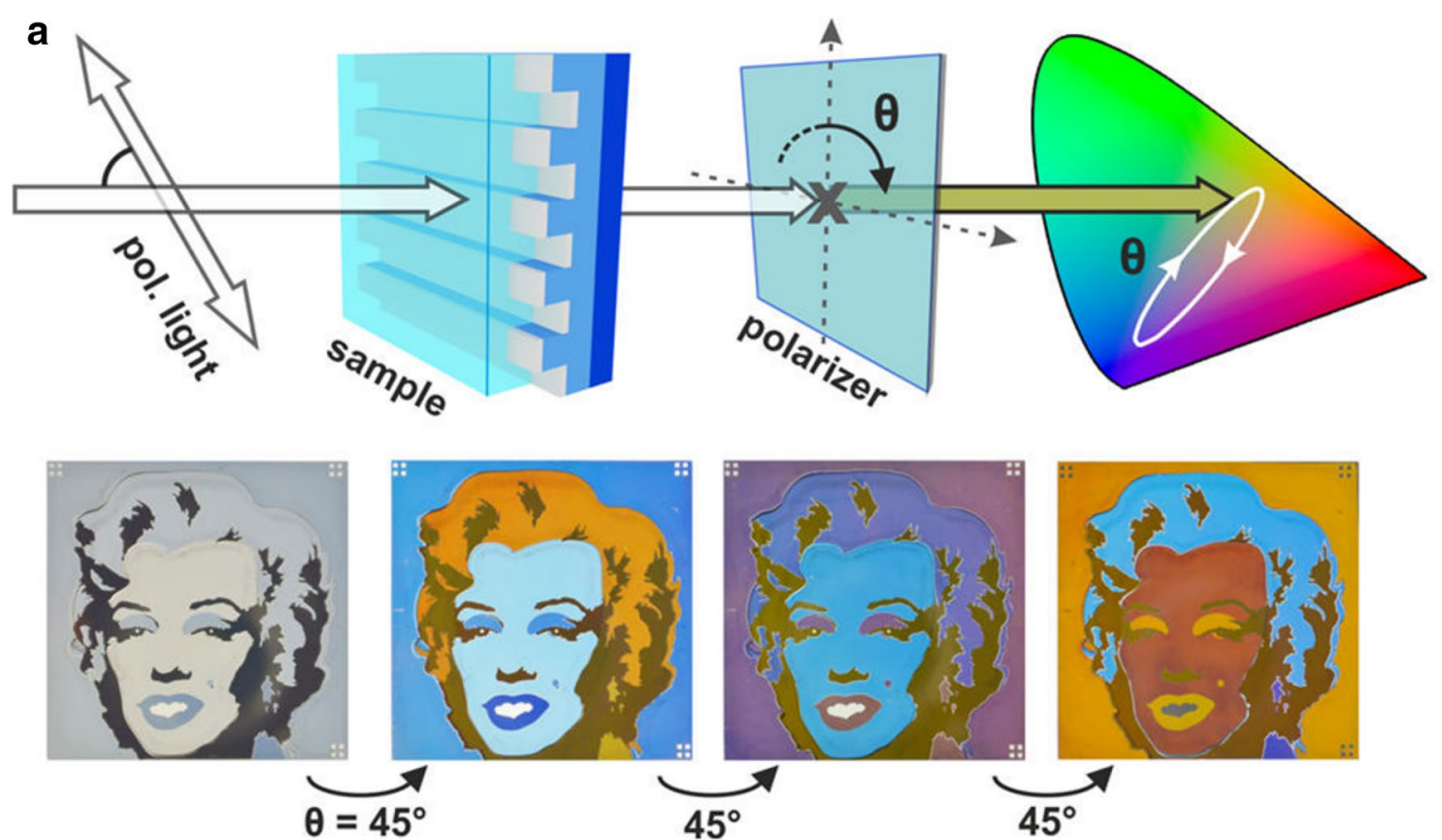

b

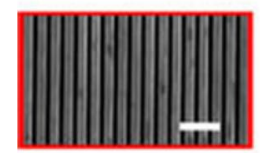

C

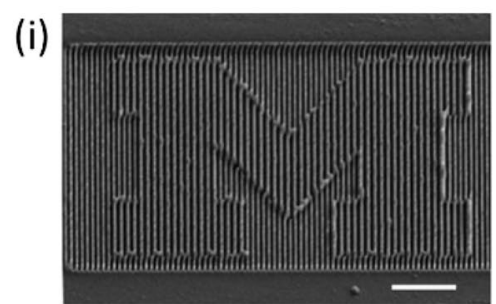

(ii)

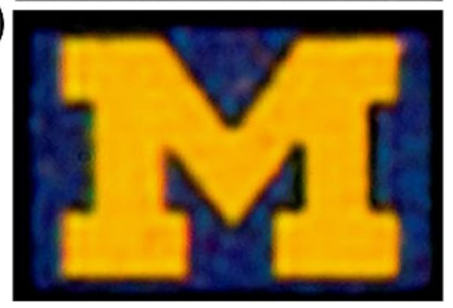

Fig. 4 a Schematic of silver-based grating color filter using plasmonic phase retarder. Four Marilyn Monroe images illustrate effects of increasing the angle of the incident polarization [91]. b Schematic design of a MIM grating coloring device. Incident white light is filtered into resonant wavelengths (colors). Inset: SEM image of a design; scale bar: $1 \mu \mathrm{m}$. c (i) SEM image of letter ' $M$ ' with 220-nm background period and 310-nm period of ' $M$ '. (ii) Optical micrograph of (i) illuminated using white light [62]

\subsection{Pure Si}

$\mathrm{Si}$ have been frequently used in printing technology because of low cost, reliability and compatibility with optoelectronic devices. Si has a high $n$, and can therefore manipulate light subwavelength scale [102, 103]. Importantly, Si particles with subwavelength size exhibit strong, optically-induced magnetic and electric Mie resonances at visible wavelengths. By exploiting this optical property, Si nanowires can be used as color filters to convert absorbed light to photocurrent [104]. Si can also efficiently tailor the symmetry of light emission and enhance magnetic radiative decay [105-109]. Many studies have used pure $\mathrm{Si}$ in a variety of geometries such as nanopillars [110] and crosses [111]. The studies mainly accomplished their goals of obtaining high-quality resonances in the entire visible range, which yield a great color gamut of high-purity colors. In devices with cross-shaped $\mathrm{Si}$ nanoresonators, the high-quality Mie resonances provide good confinement of energy to the structure [111]. 

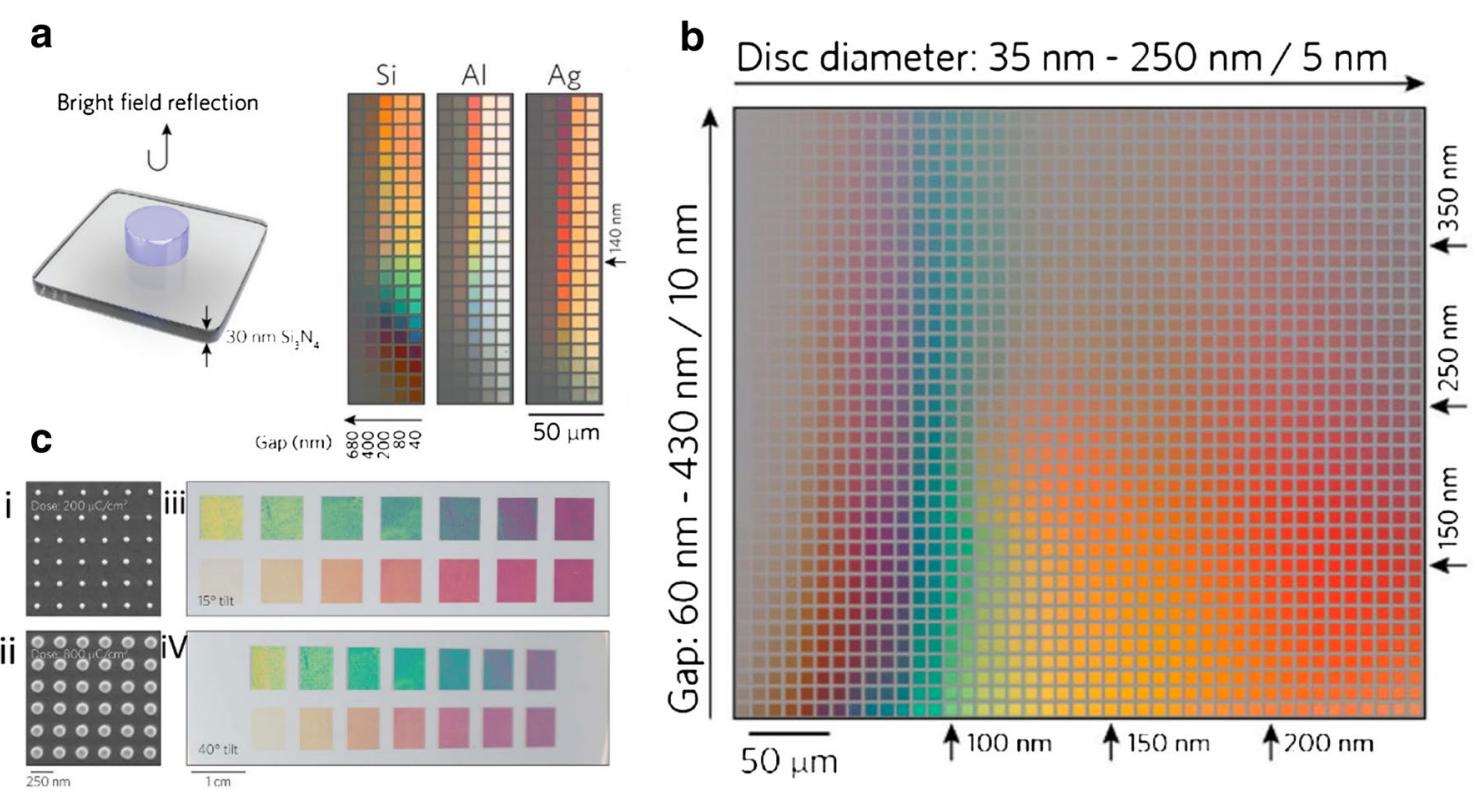

d
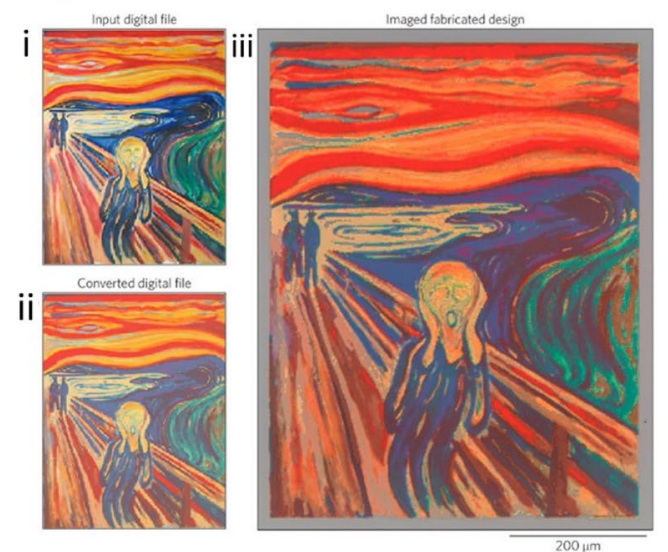

e

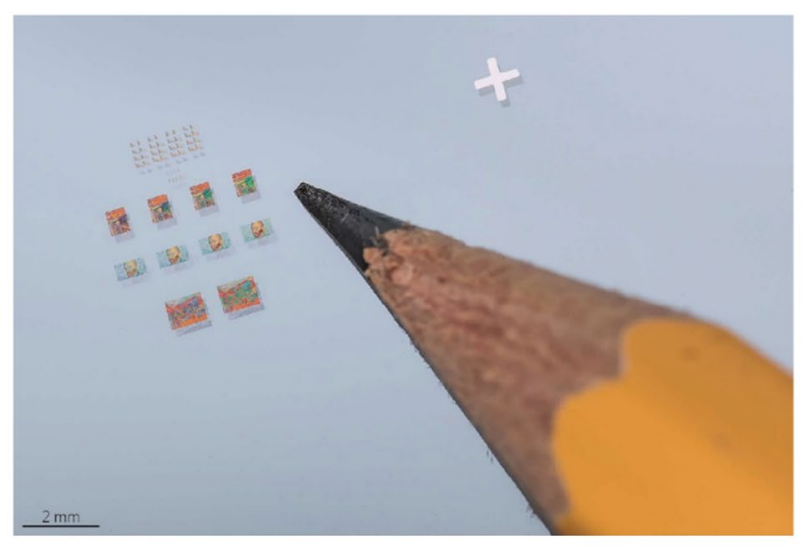

Fig. 5 a Schematic configuration of nanodisk fabricated on $\mathrm{Si}_{3} \mathrm{~N}_{4}$, with optical micrographs of $\mathrm{Si}$, Al and Ag. b Optical micrographs of a color filter using Si-based nanodisks (diameters 35-250 nm in increments of $5 \mathrm{~nm}$; gaps between nanodisks are 60-430 nm in increments of $10 \mathrm{~nm}$ ). c SEM images of dot array with a pitch of $250 \mathrm{~nm}$, and dose of (i) $200 \mu \mathrm{C} / \mathrm{cm}^{2}$ and (ii) $800 \mu \mathrm{C} / \mathrm{cm}^{2}$. Square color images $\left(7.5 \times 7.5 \mathrm{~mm}\right.$ ) tilted at (iii) $15^{\circ}$ and (iv) $40^{\circ}$. d (i) A original painting (The scream by Edvard Munch); (ii) the painting after discretization of the colors generated in (i) of $\mathbf{b}$. (iii) Optical micrograph reproduced by the color filter based on Si nanodisk $(545 \times 700 \mu \mathrm{m})$. e Photograph of reproduced paintings on a quartz wafer with a pencil for scale [112]

Research using pure Si nanodisks obtained colors and spectra that are distinct from those obtained using metal (Al, Ag) nanodisks [112] (Fig. 5a). Si provided monotonous hue variations, whereas metals exhibit rapid color changes. These differences occur because peaks are clearer in Si spectra than in $\mathrm{Al}$ and $\mathrm{Ag}$. Colors and their intensity produced by the DCFs can be adjusted by tuning the diameter and gap of nanodisks (Fig. 5b). This color exhibits little angle dependence (Fig. 5c). A reproduction of a painting replicated the original well (Fig. 5d).

\subsection{Enhanced Si and others}

To enhance the optical properties of $\mathrm{Si}$, many researchers have attempted to find improved materials and designs. Germanium (Ge) has been evaluated as a substitute for $\mathrm{Si}$ (Fig. 6a) [113]. Ge does not have high-quality resonance due to high absorption in the visible range, and therefore cannot be used alone as a color filter. A material composed of $\mathrm{Si}(0.8)$ and $\mathrm{Ge}(0.2)$ achieved higher refractive index and lower absorption coefficient than pure $\mathrm{Si}$ in most of the visible region. The ratio of Ge must be minimized to avoid high absorption. 

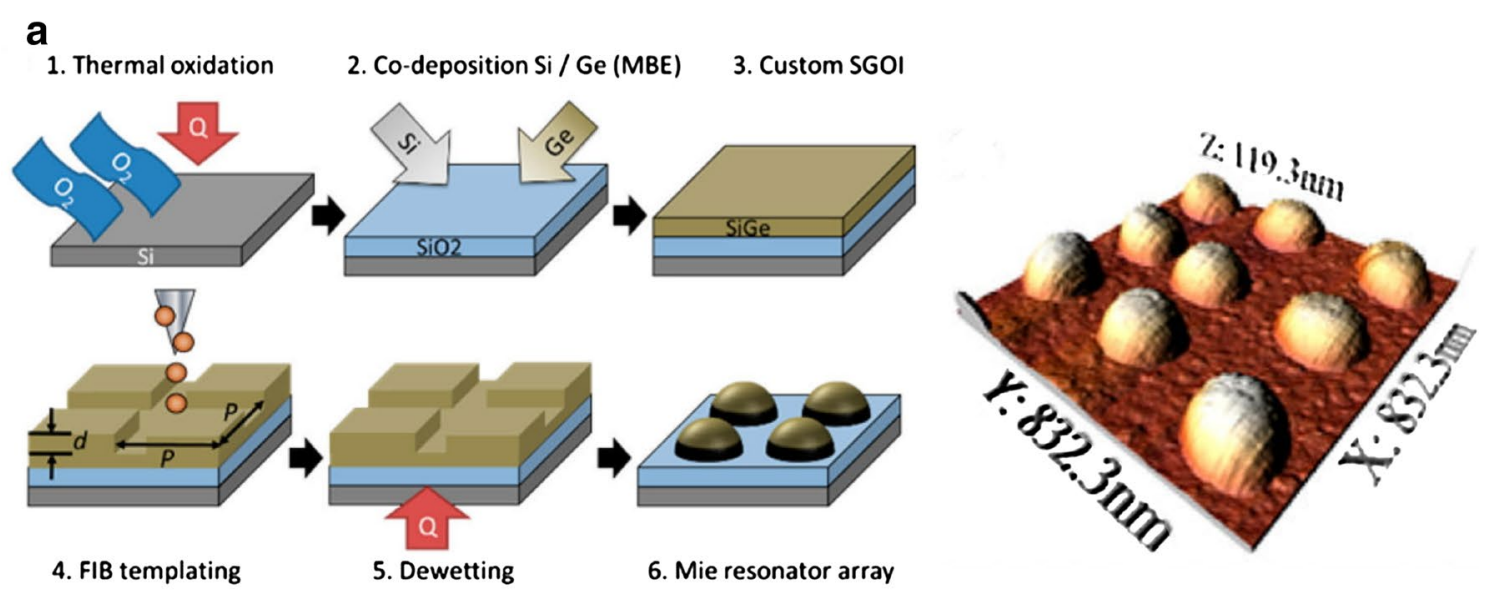

b
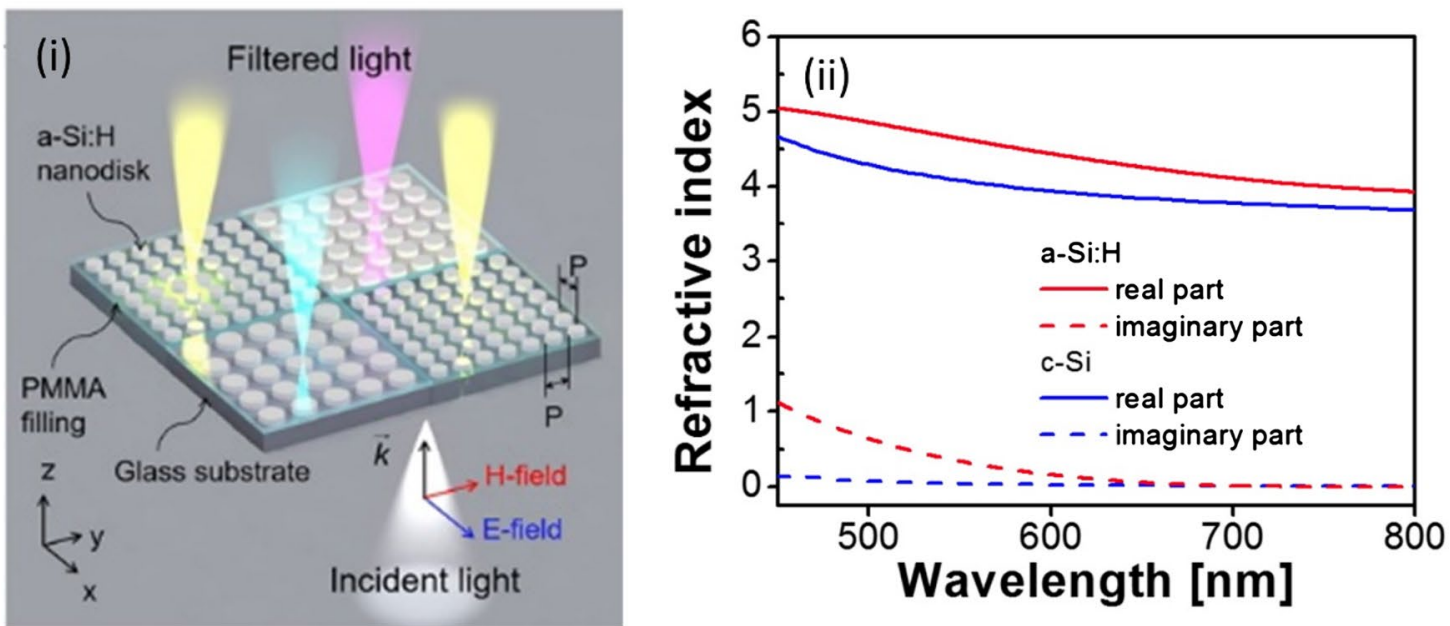

C

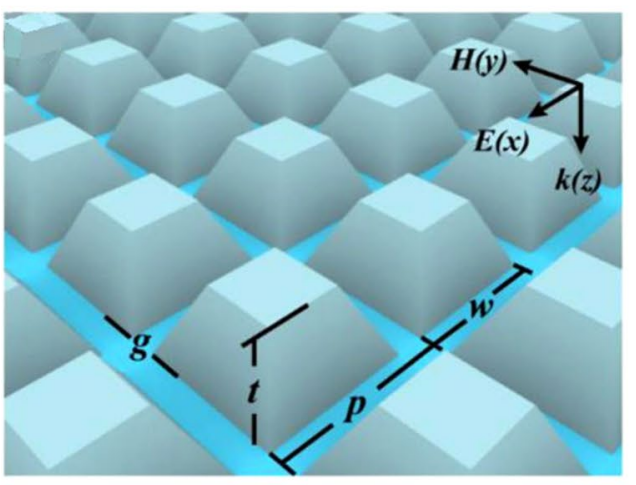

d

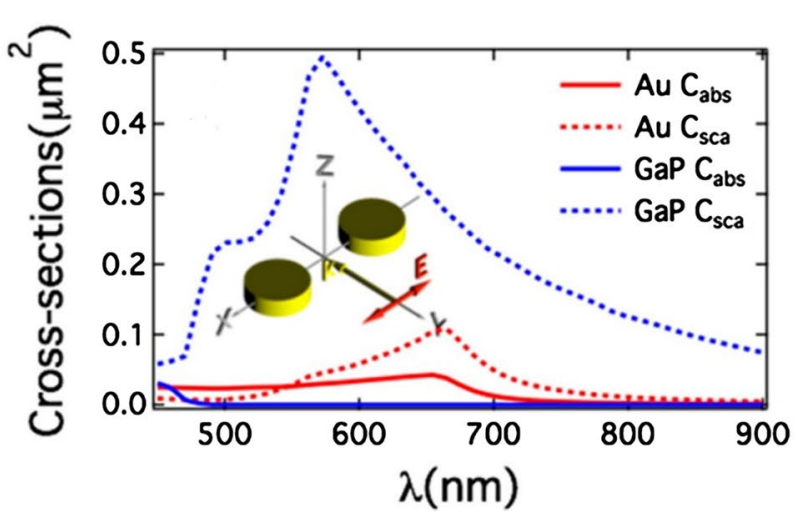

Fig. 6 a Schematic fabrication steps and AFM image of a dielectric nanoresonator [113]. b (i) Schematic design of a structural color filters exploiting amorphous Si:H nanodisks with different diameters D and period P. (ii) Refractive indices of the amorphous Si:H and crystalline Si:H [28]. c Tilted image of $\mathrm{aTiO}_{2}$-based metasurfaces [125]. d Cross sections of absorption and scattering in case of Au dimer and GaP dimer with disk shape. The disk radii are $50 \mathrm{~nm}(\mathrm{Au})$ and $100 \mathrm{~nm}(\mathrm{GaP})$ and heights are $37.5 \mathrm{~nm}(\mathrm{Au})$ and $75 \mathrm{~nm}(\mathrm{GaP})$ [126] 
Hydrogenated amorphous $\mathrm{Si}(\mathrm{a}-\mathrm{Si}: \mathrm{H})$ has been evaluated as the material in all-dielectric color filters (Fig. 6b) [28]. Many of existing all-dielectric structural filters consist of crystalline $\mathrm{Si}$ (c-Si) [10, 114-120], but they have low transmission and a challenge to grow high-quality c-Si on foreign substrates. Compared to c-Si, a-Si:H has advantages of low cost, compatibility with complementary metal-oxide-semiconductor process, and efficient growth on foreign substrates at low temperature to achieve high refractive index. The structural filter based on a-Si:H had a higher refractive index than $\mathrm{c}-\mathrm{Si}$ (Fig. 6bii). Although this design has some losses at short visible wavelengths, a-Si:H has superior optical properties, low cost, and simple fabrication, and therefore may be an alternative to other color filters.

Various materials such as $\mathrm{TiO}_{2}$ and $\mathrm{GaP}$ have been evaluated as alternatives to PCFs and other structural color filters $[38,121-124]$. $\mathrm{TiO}_{2}$ is a reasonable candidate; a recent report (Fig. 6c) obtained a suitable $n \sim 2.54$ at $400 \mathrm{~nm}$, with near-zero extinction coefficient, which means remarkably low loss in the visible range [125]. GaP has also merits in designing all-dielectric metamaterials. The scattering cross section of $\mathrm{GaP}$ is $\sim 0.5$ whereas it is $\sim 0.1$ in $\mathrm{Au}$ disks. The $\mathrm{GaP}$ absorption cross section is nearly zero from $500 \mathrm{~nm}$ on (Fig. 6d) [126]; the goal of this study was to discover a material that does not suffer from the visible-spectrum losses of PCFs through the comparison with metal (Au). Results may provide a good alternative to metals that exceeds their far-field and nearfield emission efficiency.

\section{Tunability and dynamic modulation of colors}

Dynamic color printing is essential for practical applications such as dynamic displays, cryptography and camouflage. Research into tunability of structural coloring is increasing, due a to desire for advanced and innovative functionalities of metasurfaces. Most previous designs could only generate one static color with fixed geometry, but color filtering with tunable function would have a diversity of applications. In this section, we introduce various type of tunable color filters (TCFs) that have studied recently. Methods used include applications of LCs [32-35, 127-129], of chemical transitions [130-133] and of mechanical transformations [36-39].

\subsection{Liquid crystals}

One method of achieving tunability is to exploit the anisotropy and rearrangement of LCs under applied voltage; this approach achieved rotating polarization of incident light at will. An electrically-tunable color filter with a LC polarization rotator has been achieved [127] by exploiting guided mode resonance with waveguide layers, and diffracted wave by an asymmetric Al pattern to govern resonance in proposed resonators. The design used $\mathrm{Al}$ nanoresonators that have an asymmetric gap distance between the resonators along $x$ and $y$ directions, and therefore respond differently to $x$-polarized and $y$-polarized light (Fig. 7a). With no voltage applied to a unit cell, polarization of incident light is rotated to $90^{\circ}$, but when applied voltage exceeds a threshold $V_{\mathrm{a}}$, polarization is maintained at $0^{\circ}$. At voltage $<V_{\mathrm{a}}, x$ and $y$ polarizations coexist. Transmission spectra by polarization control with voltage manipulation is expressed as $T_{\theta}=T_{0}(\lambda) \cos$ ${ }^{2} \theta+T_{90}(\lambda) \sin ^{2} \theta$ where $T_{0}$ and $T_{90}$ are the transmissions of $x$ and $y$ polarization, and $\theta$ is polarization angle. Consequently, the proposed visible dichroic resonator can be used to tune expressed color in a narrow range of CIE 1964 color coordinates (Fig. 7b).

Tunable color generation can be achieved using an imprinted structure in contacted with LC [128]. This method achieves color tunability by dynamic refractive index tuning by topological reorientation of LC. A shallow imprinted $\mathrm{Al}$ layer is surrounded by a high-birefringence LC (Fig. 7c). As unpolarized white light passes through the LC layer, the light couples to plasmonic modes at the imprinted metal surface. The orientation of the LC determines the spectral location of SPR, because SPR Modes depend on the dielectric constant of the surroundings. The LC's high birefringence causes a large plasmonic shift that leads to high range of color tunability. With no applied electric field, the LC aligns parallel to the Al surface. When an electric field is applied, the LC near the imprinted surface assumes the orientation state that minimizes its internal energy. As the voltage of the electrical field is increased, the LCs keep changing their orientation until they are all normal to the surface. This method achieves higher dpi than a conventional display, and has millisecond-scale response times (Fig. 7d). This research demonstrates the benefits of the LC-plasmonic system, and suggests a method to achieve TCFs.

This tunable device composed of imprinted structure with LC has been shown to be compatible with thin-filmtransistor (TFT) technology [129]. The imprinted plasmonic surface was surrounded by a highly birefringent LC and stacked, followed by rubbed amide, ITO, and a supersubstrate layer (Fig. 7e), and the stacked layers were integrated with a TFT array (Fig. 7f). The integrated device is connected to computer so that individual pixels are manipulated via images that the monitor displays. The authors also generated full images and a video of text editing (Fig. 7f, bottom). Although prototype had demerits including image degradation by white reflection from TFT metal lines, and inability to source high voltage, this LC-plasmonic devices shows the possibility of replacing conventional displays. 


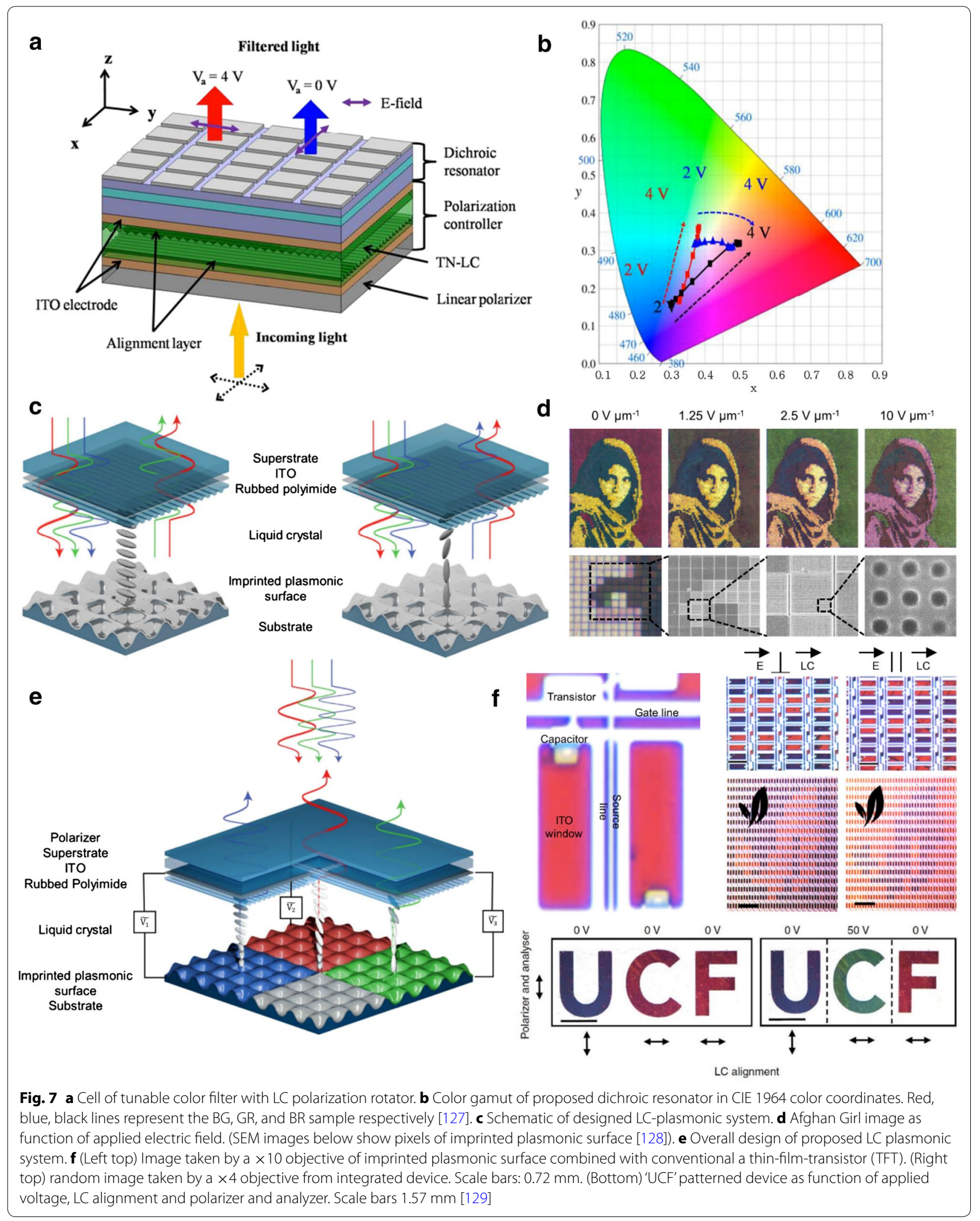



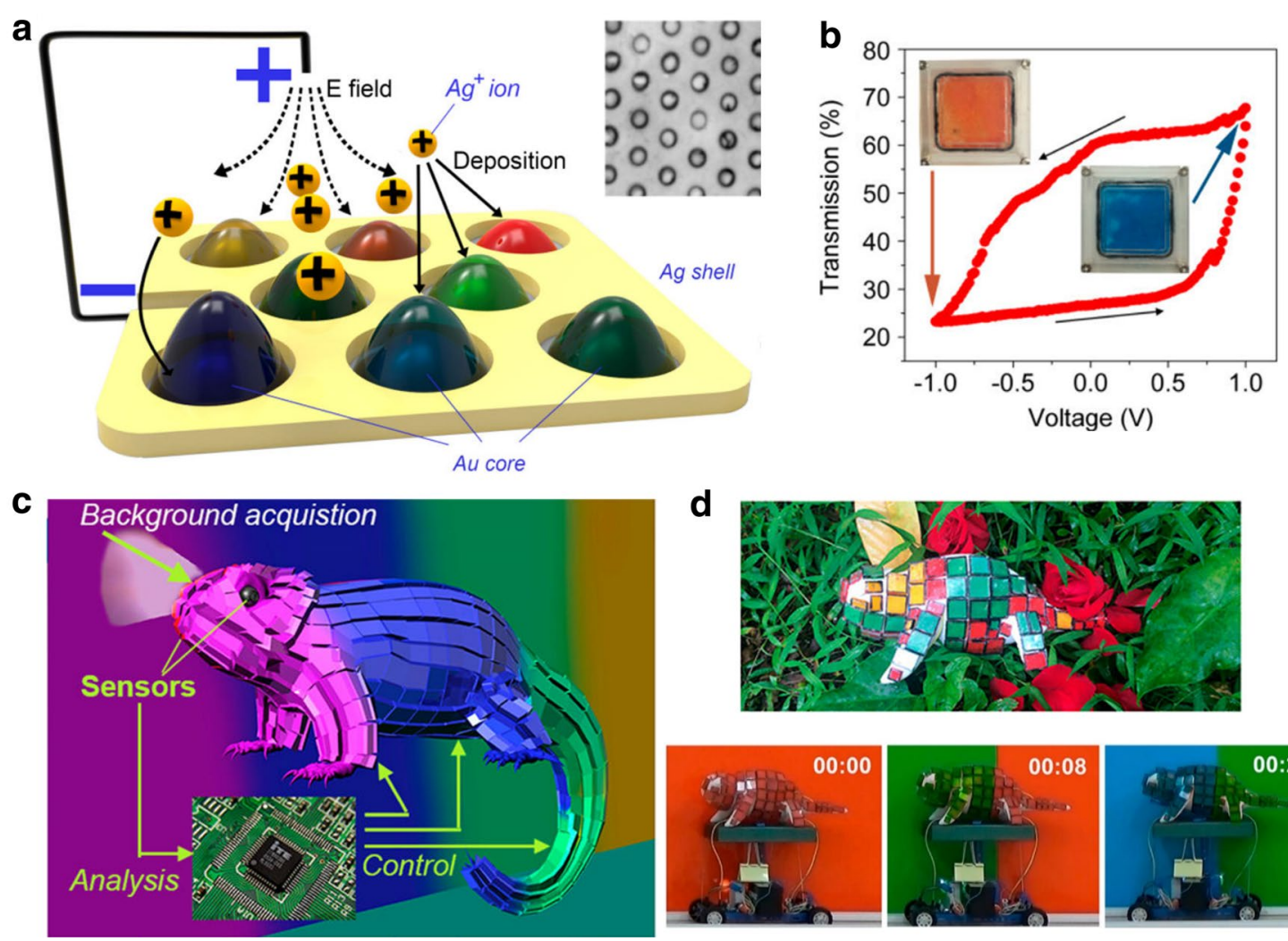

d
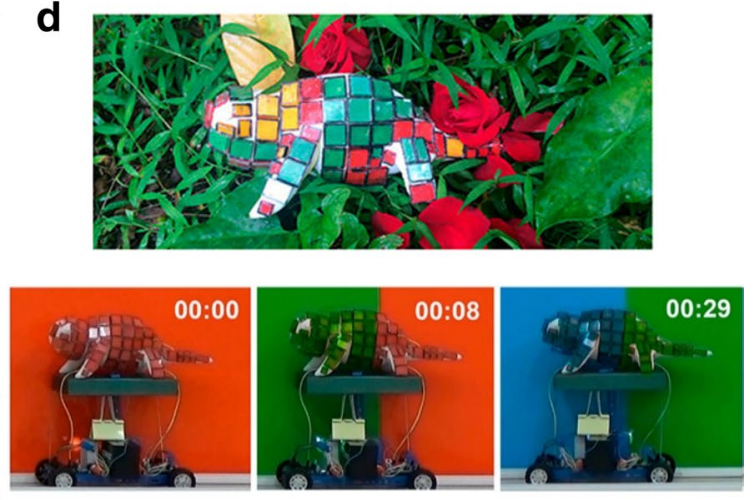

Fig. 8 a Schematic diagram of plasmonic cell. Inset: SEM image of the Au nanoshell array. b Transmission change of unit cell in response to applied voltage. Insets: photos of the cell device points marked by arrows. c Schematic of biomimetic camouflage technique obtained using the proposed design. d (Top) disguised plasmonic chameleon on outdoor grass. (Bottom) still photographs of camouflage demonstration video [130]

\subsection{Chemical transition}

Dynamic plasmonic-nanostructures formed by electrodepositing and electrostripping were evaluated as biomimetic camouflage [130]. The device exploited electrodeposition-induced color transformability of $\mathrm{Au} / \mathrm{Ag}$ core-shell nanostructures. The plasmonic arrays were composed of a bottom indium tin oxide (ITO) layer, and a perforated $\mathrm{SiO}_{2}$ layer with $\mathrm{Au} / \mathrm{Ag}$ core shells embedded in the holes (Fig. 8a). A plasmonic unit cell is produced by packaging the arrays into a device and filled with gel electrolyte that contain $\mathrm{Ag}^{+}$ions. The coloring process exploits two phenomena: the physical configuration of the plasmonic core shells is tuned by applying a voltage, and the redox reaction of $\mathrm{Ag}$ is hysteretic (Fig. 8b). As Ag deposition times increase, the nanodomes inside holes grow, and as a result the cells change color. The plasmonic cells achieved dynamic camouflage (Fig. 8c, d). The plasmonic cells were attached to the body of a mechanical chameleon that was equipped with miniature color sensors. The information from color sensors was analyzed and sent to each cell, and the colors of the chameleon were altered to match the acquired signals. The plasmonic cell device was also fabricated on a flexible substrate; this success shows the possibility of wearable dynamic camouflage.

Catalytic magnesium ( $\mathrm{Mg}$ ) metasurfaces can be used as dynamic plasmonic color displays [131]. Plasmonic colors were printed, tuned, erased and restored by exploiting time-dependent hydrogenation and dehydrogenation of Mg NPs. When placed between a titanium (Ti) adhesion layer and a Ti/palladium (Pd) complex and exposed to hydrogen, Mg NPs absorb 7.6 wt\% hydrogen and is changed from a metal to a dielectric $\left(\mathrm{MgH}_{2}\right)$ (Fig. 9a). The Mg NPs lose their plasmonic features, and reflectance spectra lose all of the features that they showed before the transition. Therefore, hydrogenation can be regarded as an erasing process; dehydrogenation can be regarded as a restoring process. These erasing and restoring processes have possible applications as optical data encryption and decryption (Fig. 9b). This new concept of plasmonic coloring has potential application such as anti-counterfeiting, optical information encryption, and animation printing. 

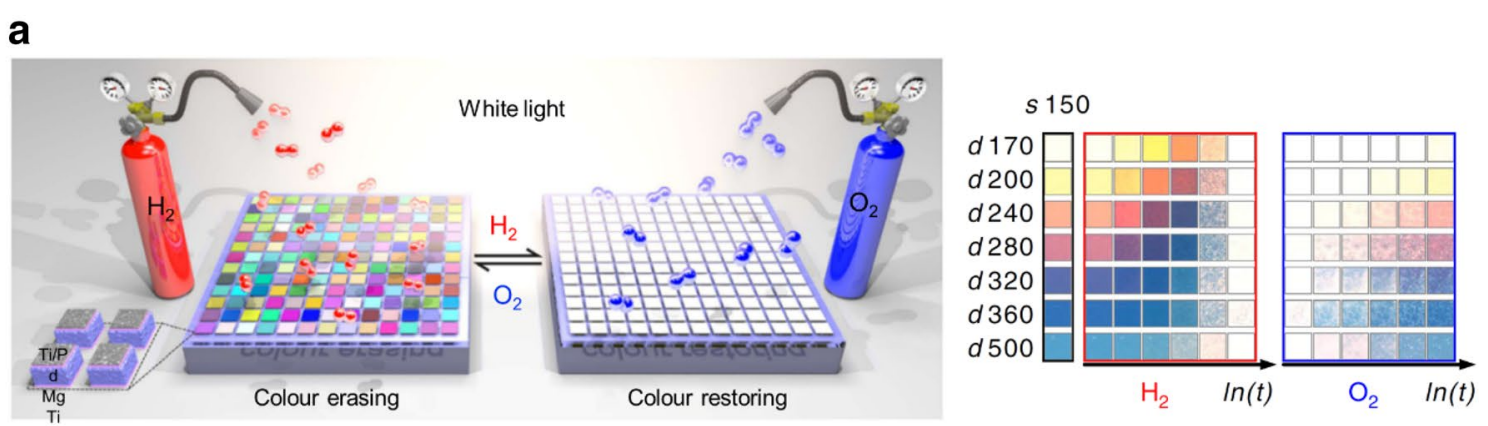

b
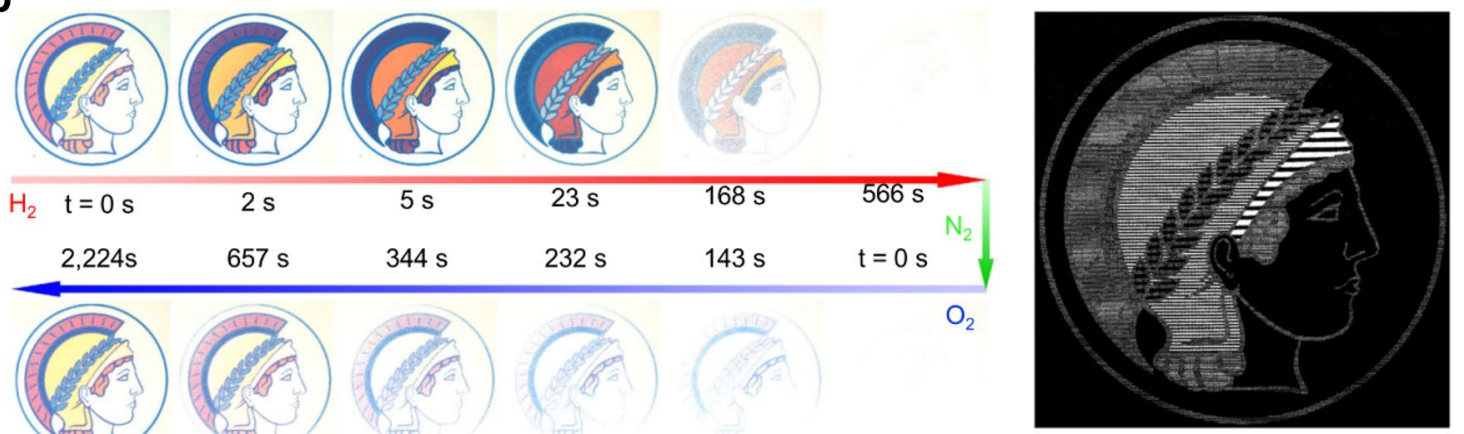

C
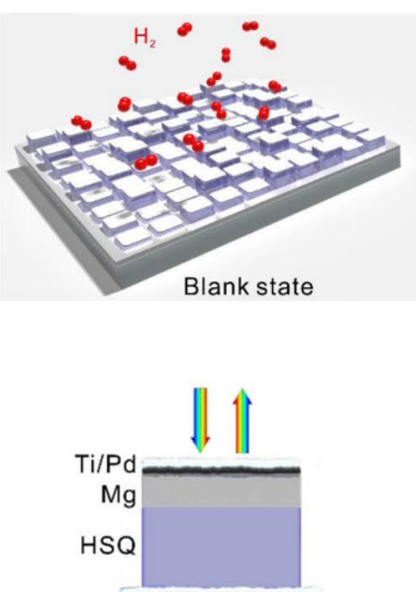

Al mirror
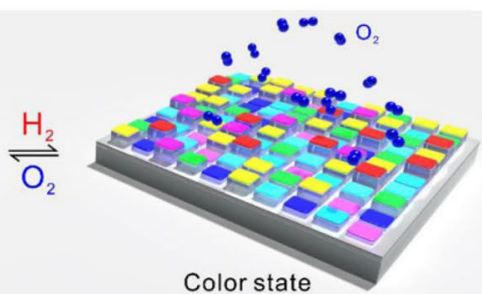

Color state

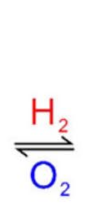

$\frac{\mathrm{H}_{2}}{\stackrel{\mathrm{O}_{2}}{\rightleftharpoons}}$

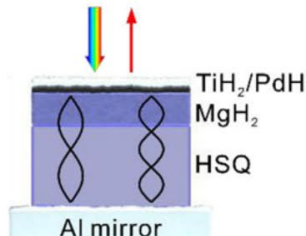

d
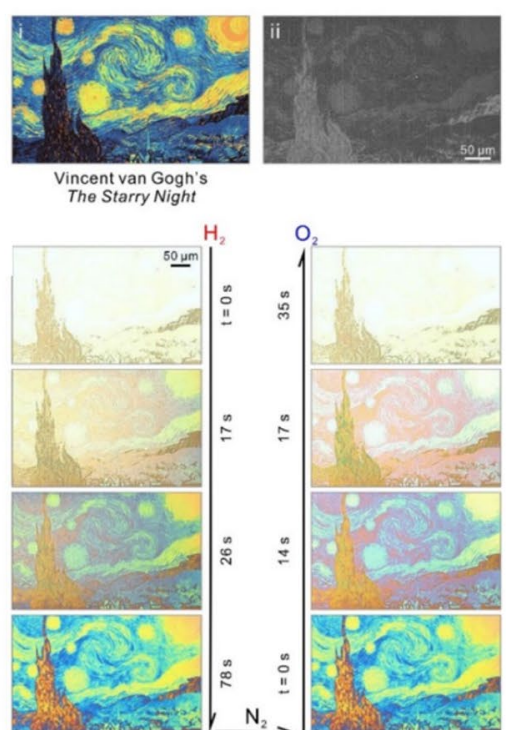

Fig. 9 a (Left) schematic diagram of plasmonic cell consisting of Mg NPs. White unpolarized light incident on the plasmonic cell in normal direction. $\mathrm{H}_{2}$ exposure erases the color; $\mathrm{O}_{2}$ restores it. (Right) color palettes as a function of $d$ and $\ln (t)$. $\mathbf{b}$ (Left) Time-varying color erasing and restoring of fabricated image. (Right) SEM image of the fabricated sample [131]. c (Top) Schematic diagram of proposed dynamic stepwise cavity resonators. Upon $\mathrm{H}_{2}$ exposure, color information is shown; $\mathrm{O}_{2}$ exposure erases the color; $\mathrm{H}_{2}$ restores it. (Bottom) cross section view of unit Fabry-Pérot resonator. Under $\mathrm{H}_{2}$, a double dielectric spacer is formed and the $\mathrm{TiH}_{2} / \mathrm{PdH}$ layer transmits incident light. $\mathbf{d}$ (Top) Vincent van Gogh's Starry Night and fabricated SEM image. (Bottom) color erasing and restoring processes as a function of $\mathrm{H}_{2}$ and $\mathrm{O}_{2}$ exposure time [132]

Dynamic displays have been achieved using FabryPerot cavity resonators that exploit this metal-to-dielectric transition of Mg [132]. Hydrogen absorption by the $\mathrm{Mg}$ layer results in state switch between metal and dielectric; hydrogen desorption causes the reverse process.
Upon hydrogenation, a capping layer is changed from $\mathrm{Ti} /$ Pd to $\mathrm{TiH}_{2} / \mathrm{PdH}$, and as a result light can pass through dielectric spacers $\left(\mathrm{MgH}_{2}+\mathrm{HSQ}\right)$ and be reflected by an $\mathrm{Al}$ mirror. Then Fabry-Perot resonance modes form in the cavity so that reflected light generates vivid colors 
(Fig. 9c, bottom, d). Under hydrogen exposure, the color changes, and reaches its final state after $78 \mathrm{~s}$. Under oxygen exposure, the image is restored to the original state within $35 \mathrm{~s}$.

\subsection{Mechanical deformation}

Tunability of metasurfaces [39] has applications and possibilities for commercialization. Mechanical deformation is one approach to achieve this tunability. Structural color can be generated by mechanical deformation [36]; specifically by stretching a photonic-crystal slab patterned on a flexible and elastic substrate. This method has achieved resonance-induced reflectance that has sharp resonance peaks; this process is related to Fano resonance [134]. Optical Fano resonance can be regarded as an interference effect between directly reflected light and modes that leak into the environment. Because the reflected light and radiated light have same phase information,
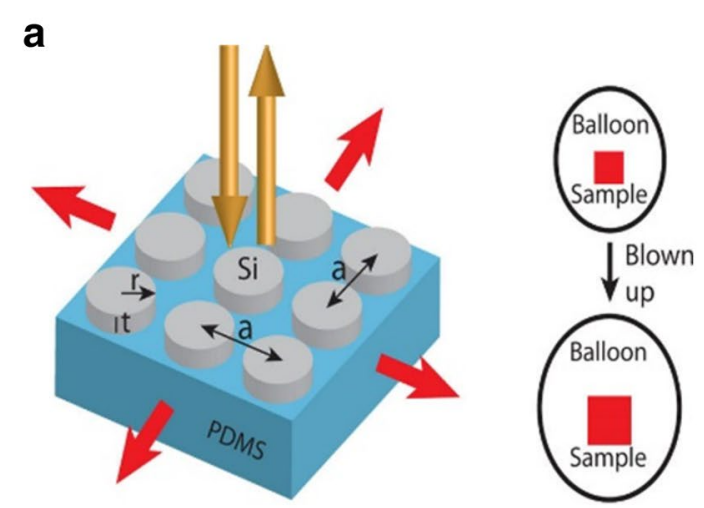

C

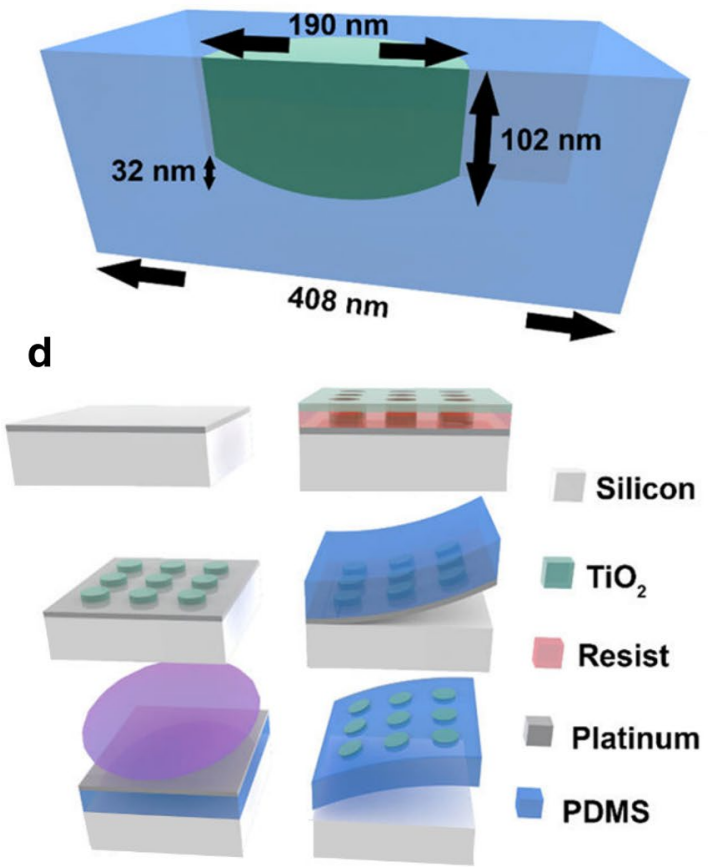

b
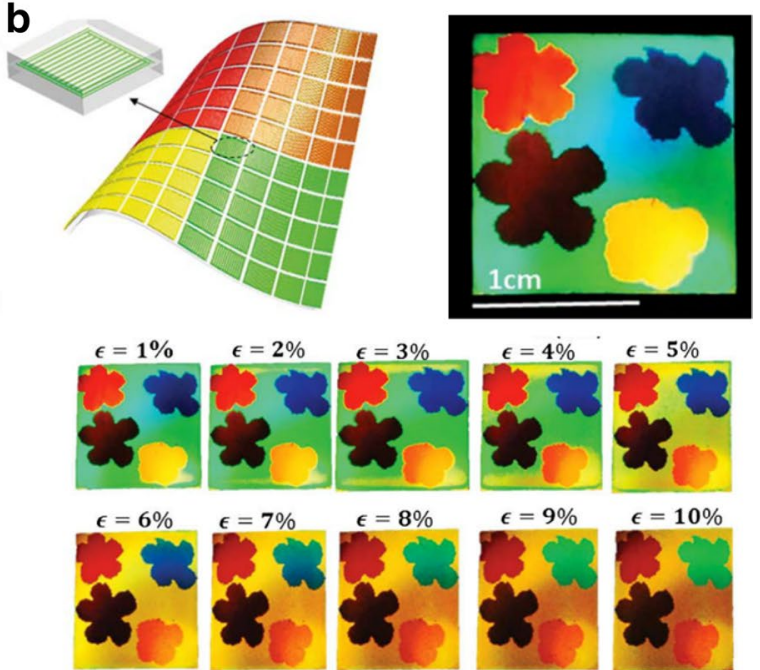

e
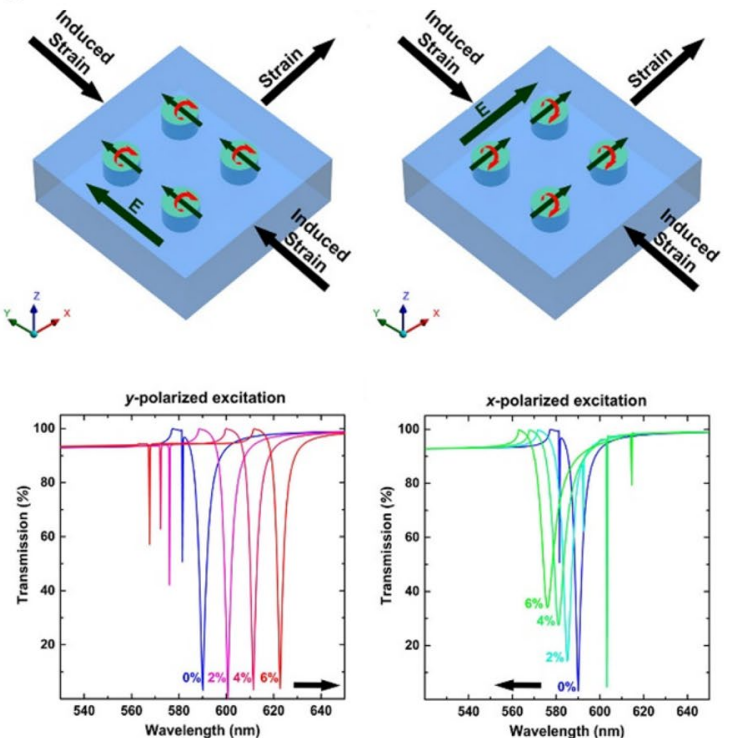

Fig. 10 a Schematic of mechanically-deformed photonic crystal slap. Inset: Illustration of deformation process [36]. b HCM designs and magnified HCM unit cells, and photo of fabricated sample with flower pattern. The scale bar $1 \mathrm{~cm}$. (Bottom) images of color-changing flower as a function of stretching $\varepsilon=\Delta \mathrm{L} / \mathrm{L}$ [37]. $\mathbf{c}$ Embedded $\mathrm{TiO}_{2}$ nanorod in PDMS. d Illustration of fabrication process. e (Top) $y$-polarized and $x$-polarized dipole orientation with respect to strain direction. (Bottom) $y$-polarized and $x$-polarized transmission spectra with respect to increasing strain. Black arrow: direction of resonance wavelength shifts [38] 
constructive interference sharpens the resonance. The reflectance peak can be controlled at will by changing the periodicity $a$ in an array of nanodots (Fig. 10a). An array of Si nanorods was patterned on polydimethylsiloxane (PDMS) substrate, then attached to a black balloon to stretch the sample isotropically. Stretching to $10 \%$ yielded 32-nm spectral shift; repeated stretching did not cause degradation.

The change in colors can be widened and improved by using a high-contrast metastructure (HCM) composed of metagrating embedded in a transparent and flexible PDMS membrane [37]. HCM pixels were patterned by deep ultraviolet step-lithography, then the Si metagrating was etched to remove the $\mathrm{SiO}_{2}$ layer. The HCM was covered by PDMS and detached from SOI Wafer. The resulting PDMS stamp was further protected by encapsulation in a second PDMS layer. Deformation from $\varepsilon=0-10 \%$, tuned the color of a flower image fabricated using the suggested structures (Fig. 10b). The HCM has good repeatability under stretching cycles, so this technology may have applications in camouflage and biolabeling.

Mechanical deformation of all-dielectric metasurfaces can also achieve tunable color at visible frequencies [38]. The design was an array of $\mathrm{TiO}_{2}$ rods embedded in a PDMS layer (Fig. 10c). The array was patterned by EBL, followed by etching. The PDMS was deposited on top of $\mathrm{TiO}_{2}$ array to form embedded $\mathrm{TiO}_{2}$ geometry (Fig. 10d). To test color tunability strain was applied the metasurfaces of $\mathrm{TiO}_{2}$ resonators in PDMS in two directions orthogonal to each other; with only $6 \%$ strain, the resonance peaks shifted $5.08 \%$ to red under $x$ polarization, and shifted $0.96 \%$ to blue under $y$ polarization (Fig. 10e, bottom).

\section{Scalable fabrication for further practical applications}

The above-mentioned structural color filters are mainly fabricated by conventional patterning methods such as EBL or focused ion beam milling. These methods seem appropriate for manufacturing subwavelength nanostructures due to an ability to fabricate them elaborately. However, these fabrication methods have limitations such as limited area, low throughput, intricate process, and high cost. In addition, the exceptional potentials and advantages of structural color filters remain a challenge to actualize in practice because of a shortage of scalable and high-speed fabrication methods. If color filtering devices can be fabricated over large area at high throughput, the technologies will have many practical applications and will be useful in major industrial fields [53, 65, 135-139]. Thus, a fabrication method that allows scaleup and fast manufacturing of nanostructures should be developed.

A laser coloring process with high throughput produces a variety of non-iridescent colors by using 'normal' and 'burst' modes with a picosecond laser [140]. The picosecond laser is chosen due to lower costs, stronger pulse energies and quicker throughput than existing femtosecond lasers. 'Normal' mode uses the laser as usual; in 'burst' mode, the same number and energies of pulses are irradiated within a much shorter time than in normal mode to reduce heat damage, increase processing speed, and increase phonon-electron coupling [141-143]. The burst coloring method achieved better quality of colors than the nonburst method (Fig. 11b). Each palette has single a parameter that corresponds to line spacing, laser scanning speed and angle between machining direction and light polarization. The $13.5-\mu \mathrm{m}$ line spacing and $210-$ $\mathrm{mm} / \mathrm{s}$ laser scanning speed at maxima prove that this approach will contribute to practical applications and commercialization of plasmonic color printing.

Bottom-up methods have widely used to achieve largearea plasmonic colors quickly [144-147]. One such approach exploits colloidal self-assembly fabrication to construct regularly patterned nanostructures with low cost on a centimeter scale [148]. Various structure shapes such as disk, dome-ring and ring can be manufactured according to different fabrication processes (Fig. 12a). To summarize the methods, self-assembled monolayer polystyrene (PS) spheres are transferred onto a spincoated HSQ layer in accordance with Langmuir-Blodgett approach $[145,146]$, then the PS spheres are reduced in size by reactive-ion etching. Al disks are obtained by additional $\mathrm{O}_{2}$ deep reactive-ion etching (DRIE) and $\mathrm{Al}$ e-beam deposition (Fig. 12a, route I). The dome-rings are obtained using short $\mathrm{O}_{2}$ DRIE and Al e-beam deposition (Fig. 12, route II). Al rings are created by ultrasonic lift-off to detach the $\mathrm{Al}$ dome. The device has many

(See figure on next page.)

Fig. 11 a (i) Photograph of a silver-based coin induced by laser with large dimensions of 21-cm diameter and 2.5-cm thickness when compared to butterfly images in detail later. (ii) Close-up image of (i) illustrating detailed coloring. Butterfly shapes on the coin (iii) before and (iv) after laser irradiation. b Color palettes at (i) nonburst mode and (ii, iii) burst mode. In (i) 25 colors were produced by varying line spacing in from 1 to $13.5 \mu \mathrm{m}$ increments of $0.5 \mu \mathrm{m}$. In (ii), the color palette was created by tuning machining speed from 40 to $210 \mathrm{~mm} / \mathrm{s}$ in increments of $5 \mathrm{~mm} / \mathrm{s}$. In (iii), the color palette is generated by changing machining angle between machining direction and polarization of light. Column A: 2 passes at $270^{\circ}$; Column B: 1 pass at $270^{\circ}$ and 1 pass at $234^{\circ} ; \mathrm{C}: 1$ pass at $270^{\circ}$ and 1 pass at $191^{\circ}$; D: 1 pass at $270^{\circ}$ and 1 pass at $162^{\circ}$; E: 1 pass at $270^{\circ}$ and 1 pass at $126^{\circ}$. Rows represent spacing between lines $1-5 \mu \mathrm{m}[140]$ 


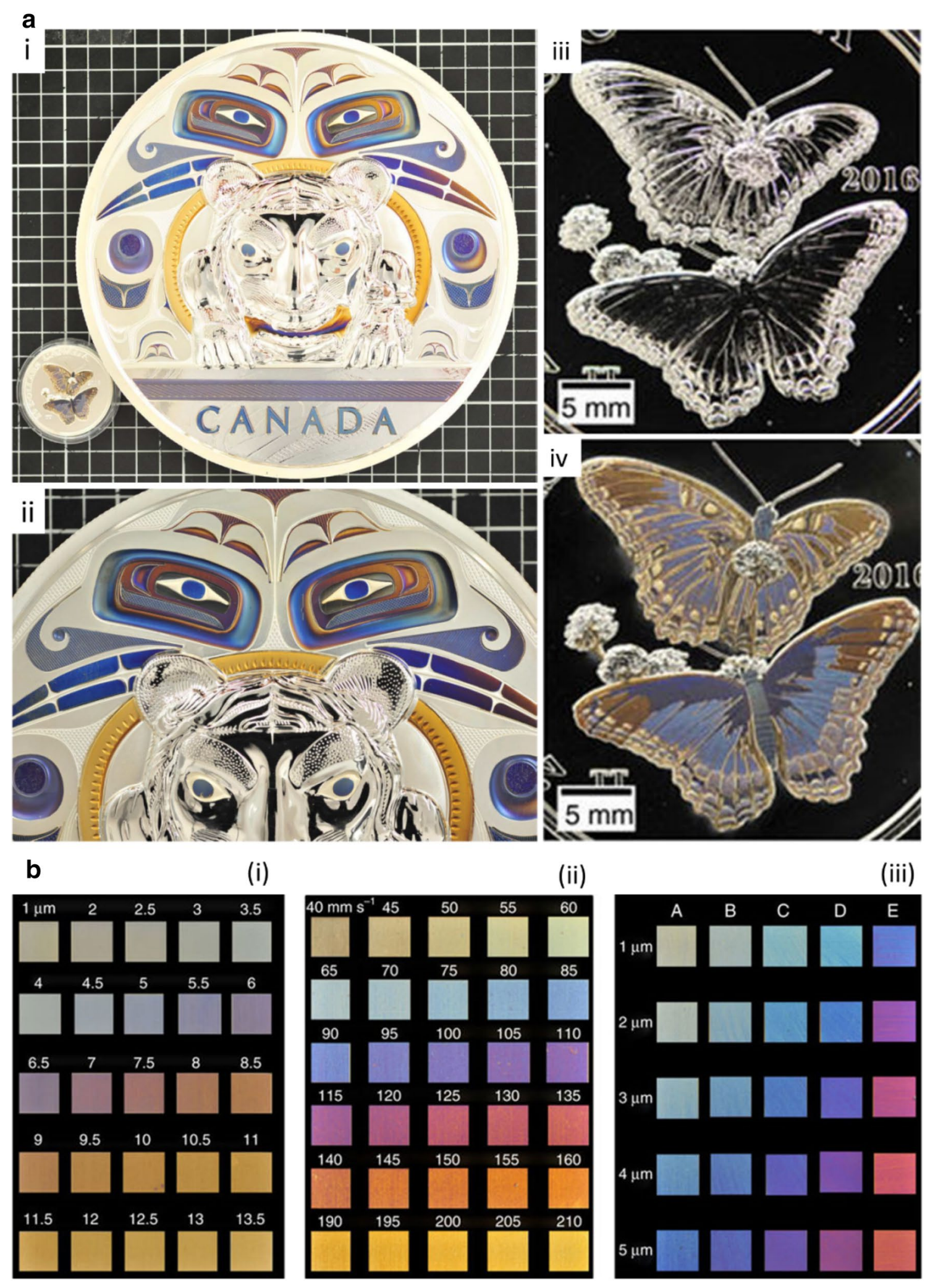




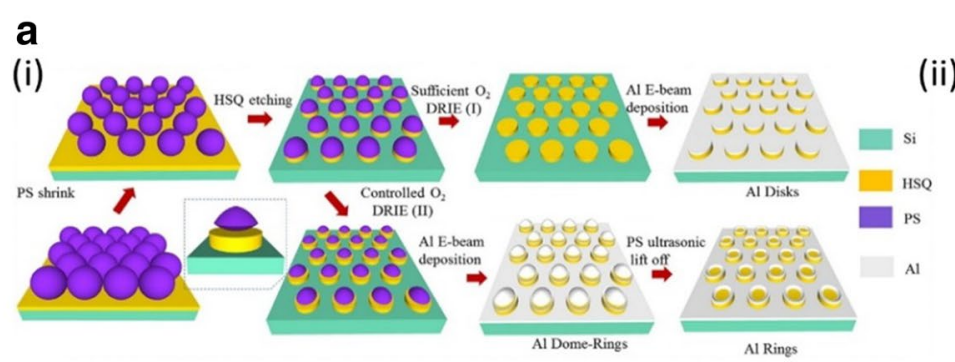

(ii)

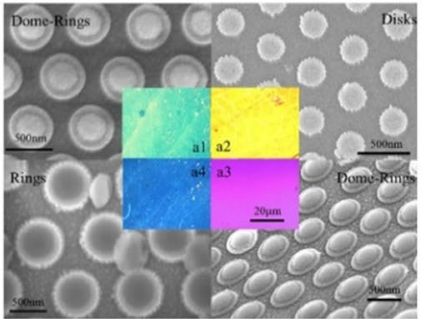

b
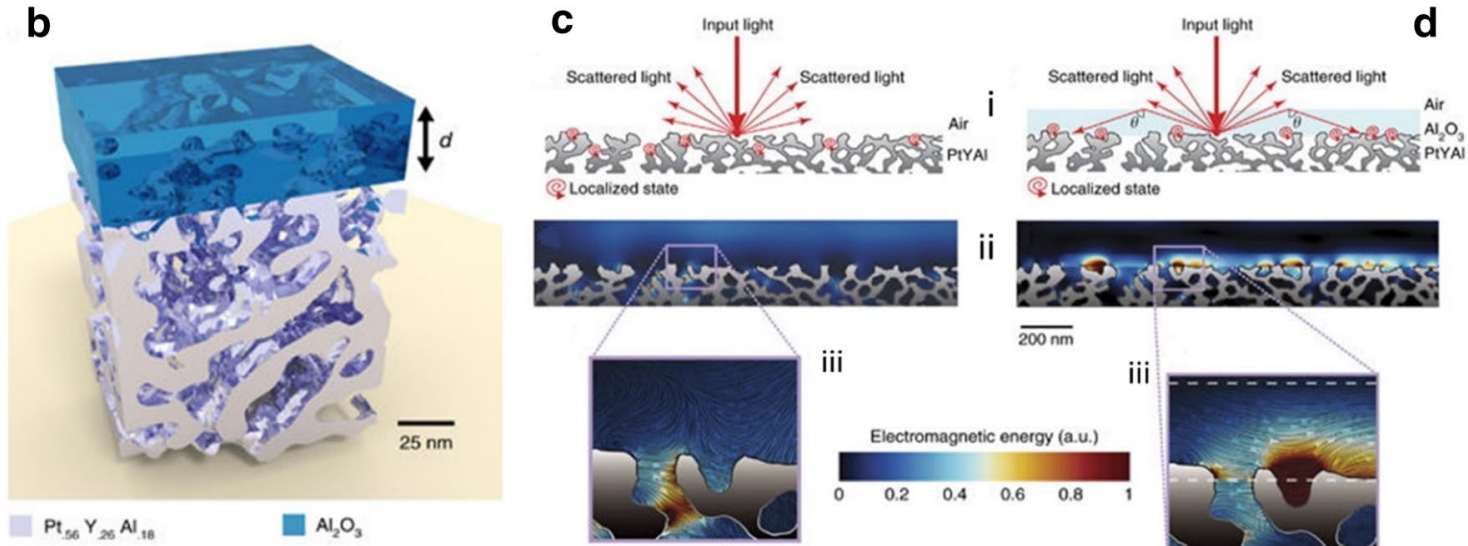

ii

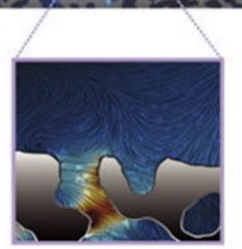

iii

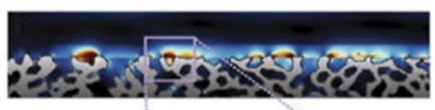

$\overline{200 \mathrm{~nm}}$

Electromagnetic energy (a.u.)
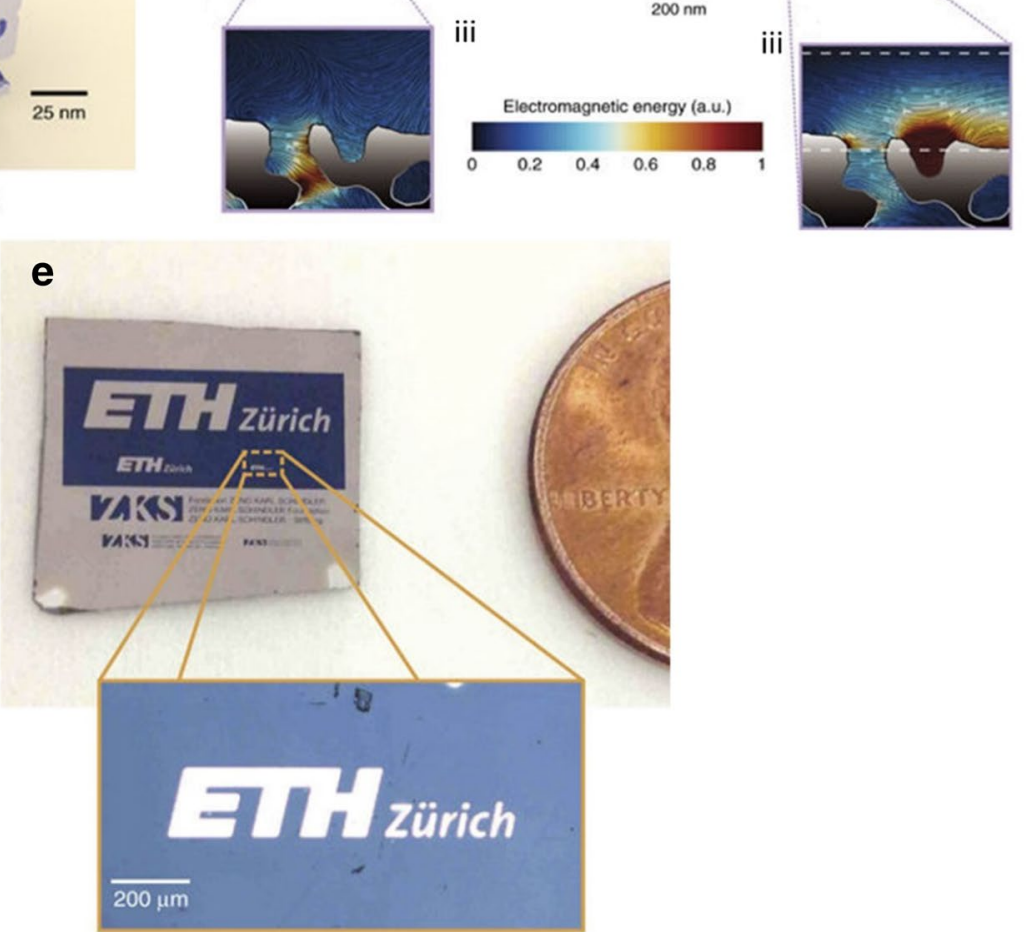

Fig. 12 a (i) Schematic flows of fabrication steps to produce disk, Al dome-ring and ring structures. (ii) SEM images of structures and corresponding color responses [148]. b Schematic design of a network metamaterials based on PtYAl and $\mathrm{Al}_{2} \mathrm{O}_{3}$ layer. c, d Working principles and calculated energy distributions of network metamaterials without $\mathbf{c}$ and with $\mathbf{d ~} \mathrm{Al}_{2} \mathrm{O}_{3}$ layer. (i) Schematic diagram of interactions between light and matter. (ii) Energy distributions in fabricated structures at $\lambda=425 \mathrm{~nm}$. (iii) Magnified images of (ii) illustrating electromagnetic energy flow indicated by arrows. $\mathbf{e}$ Photograph of a graphic design based on the network metamaterials. Inset: optical microscopic image showing two uniform colors [149]

geometric parameters that can be adjusted to tune reflection, such as diameter, height, pitch and width of structures. The device obtained high saturation in the blue region, but low saturation in the green-to-red region. The colors could be adjusted by varying the geometric parameters (Fig. 12a, samples a1-a4). This approach can fabricate 20 samples of $1.5 \times 1.5 \mathrm{~cm}$ in $<3 \mathrm{~h}$, so it seems suitable for fast mass production.

A novel optical nanomaterial based on large-scale network metasurfaces forms vibrant structural colors varied from thickness of an ultra-thin alumina coating (Fig. 12b) [149]. This approach has biomimetic 
optical properties inspired by a bird, Cotinga maynana, which has blue feathers that are iridescent in a way that cannot be explained by Rayleigh or Mie scattering. A dielectric coating reflects scattered waves to increase scattering, hence generating electromagnetic energy flow and resonant coupling in an $\mathrm{Al}_{2} \mathrm{O}_{3}$ layer (Fig. 12c, d). Color responses and resonant reflectance can be adjusted by modulating the coating thickness, and are blue-shifted as the thickness of the dielectric is increased. The device had high mechanical resistance in a scratch test. A last illustration of figures demonstrates that this approach is compatible with large-area production (Fig. 12e).

\section{Conclusion and outlook}

We have reviewed recent progress in resonance-assisted color generation. Colors achieved using plasmonic resonance and Mie resonance have intriguing features such as exceedingly high resolution, near-permanent lifetime and material simplicity. The resolution may exceed $10^{5} \mathrm{dpi}$, which surpasses the diffraction limit of light. Resonanceassisted coloring requires only a single or a few nano-size layers, so processing conditions are simple. However, the method has high patterning costs, low throughput and elaborate color tuning mechanisms; these disadvantages must be overcome before commercial applications are possible. Nanoimprinting, self-assembly and laser printing are possible solutions to achieve large-area fabrication and high throughput. LCs, chemical transition and Mechanical deformation may enable accurate and easy color tuning process.

To summarize, resonance-based color printing methods are advancing toward to real-world application. Their features such as ultrahigh resolution, brilliant optical response and compatibility with existing fabrication technologies seem to show a promising future. If fabrication costs can be reduced, and color tuning mechanisms can be improved, these methods will have important potential applications in cryptography, security, imaging, optical data storage and further optical devices.

\footnotetext{
Abbreviation

Ag: silver; Al: aluminium; $\mathrm{Al}_{2} \mathrm{O}_{3}$ : alumina; $\mathrm{Au}$ : gold; a-Si:H: hydrogenated amorphous silicon; c-Si: crystalline silicon; DCFs: dielectric-based color filters; DRIE: deep reactive-ion etching; EBL: electron beam lithography; EOT: extraordinary optical transmission; Ge: germanium; HCM: high-contrast metastructure; HSQ: hydrogen silsesquioxane; ITO: indium tin oxide; LA: long arm; LC: liquid crystal; Mg: magnesium; MIM: metal-insulator-metal; NPs: nanoparticles; PCFs: plasmonic color filters; Pd: palladium; PDMS: polydimethylsiloxane; PS: polystyrene; SA: short arm; SEM: scanning electron microscope; Si: silicon; SP: surface plasmon; SPRs: surface plasmon resonances; SPPs: surface plasmon polaritons; TCFs: tunable color filters; TFT: thin-film transistor; Ti: titanium; $\mathrm{TiO}_{2}$ titanium dioxide.
}

\section{Authors' contributions}

$\mathrm{TL}$ and $\mathrm{JJ}$ contributed equally to writing the manuscript. $\mathrm{HJ}$ helped writing the manuscript. JR guided manuscript preparation. All authors read and approved the final manuscript.

\section{Author details}

${ }^{1}$ Department of Mechanical Engineering, Pohang University of Science and Technology (POSTECH), Pohang 37673, Republic of Korea. ${ }^{2}$ Department of Chemical Engineering, Pohang University of Science and Technology (POSTECH), Pohang 37673, Republic of Korea.

\section{Acknowledgements}

The authors thank Gwanho Yoon (POSTECH) for the fruitful discussion.

\section{Competing interests}

The authors declare that they have no competing interests.

\section{Funding}

This work is financially supported by the National Research Foundation Grants (NRF-2017R1E1A1A03070501, NRF-2015R1A5A1037668 and CAMM2014M3A6B3063708) funded by the Ministry of Science, ICT and Future Planning (MSIP) of the Korean government. JJ acknowledges Hyundai Motor Chung Mong-Koo Fellowship.

Ethics approval and consent to participate Not applicable.

\section{Publisher's Note}

Springer Nature remains neutral with regard to jurisdictional claims in published maps and institutional affiliations.

Received: 27 November 2017 Accepted: 26 December 2017

Published online: 10 January 2018

\section{References}

1. T.S. Kustandi, H.Y. Low, J.H. Teng, I. Rodriguez, R. Yin, Mimicking dominolike photonic nanostructures on butterfly wings. Small 5, 574-578 (2009)

2. J. Huang, X. Wang, Z.L. Wang, Controlled replication of butterfly wings for achieving tunable photonic properties. Nano Lett. 6, 2325-2331 (2006)

3. T.-H. Wong, M.C. Gupta, B. Robins, T.L. Levendusky, Color generation in butte y wings and fabrication of such structures. Opt. Lett. $\mathbf{2 8}$, 2342-2344 (2003)

4. C. Lawrence, P. Vukusic, R. Sambles, Grazing-incidence iridescence from a butterfly wing. Appl. Opt. 41, 437-441 (2002)

5. M. Srinivasarao, Nano-optics in the biological world: beetles, butterflies, birds, and moths. Chem. Rev. 99, 1935-1961 (1999)

6. H. Ghiradella, Light and color on the wing: structural colors in butterflies and moths. Appl. Opt. 30, 3492-3500 (1991)

7. S. Kinoshita, S. Yoshioka, J. Miyazaki, Physics of structural colors. Rep. Prog. Phys. 71, 076401 (2008)

8. P. Vukusic, J.R. Sambles, Photonic structures in biology. Nature $\mathbf{4 2 4}$ 852-855 (2003)

9. K. Seo, M. Wober, P. Steinvurzel, E. Schonbrun, Y. Dan, T. Ellenbogen, K.B. Crozier, Multicolored vertical silicon nanowires. Nano Lett. 11 1851-1856 (2011)

10. H. Park, Y. Dan, K. Seo, Y.J. Yu, P.K. Duane, M. Wober, K.B. Crozier, Filter-free image sensor pixels comprising silicon nanowires with selective color absorption. Nano Lett. 14, 1804-1809 (2014)

11. V.R. Shrestha, S.S. Lee, E.S. Kim, D.Y. Choi, Non-iridescent transmissive structural color filter featuring highly efficient transmission and high excitation purity. Sci. Rep. 4, 4921 (2014)

12. J.A. Schuller, E.S. Barnard, W. Cai, Y.C. Jun, J.S. White, M.L. Brongersma, Plasmonics for extreme light concentration and manipulation. Nat. Mater $9,193-204$ (2010) 
13. D.K. Gramotnev, S.I. Bozhevolnyi, Plasmonics beyond the diffraction limit. Nat. Photonics 4, 83-91 (2010)

14. X. Chen, L. Huang, H. Mühlenbernd, G. Li, B. Bai, Q. Tan, G. Jin, C.W. Qiu, S. Zhang, T. Zentgraf, Dual-polarity plasmonic metalens for visible light. Nat. Commun. 3, 1198 (2012)

15. T.W. Ebbesen, H.J. Lezec, H.F. Ghaemi, T. Thio, P.A. Wolff, Extraordinary optical transmission through sub-wavelength hole arrays. Nature $\mathbf{3 9 1}$ 667-669 (1998)

16. N. Yu, F. Aieta, P. Genevet, M.A. Kats, Z. Gaburro, F. Capasso, A. Broadband, background-free quarter-wave plate based on plasmonic metasurfaces. Nano Lett. 12, 6328-6333 (2012)

17. W.T. Chen, K.Y. Yang, C.M. Wang, Y.W. Huang, G. Sun, I.D. Chiang, C.Y. Liao, W.L. Hsu, H.T. Lin, S. Sun, L. Zhou, A.Q. Liu, D.P. Tsai, High-efficiency broadband meta-hologram with polarization-controlled dual images. Nano Lett. 14, 225-230 (2014)

18. G. Li, M. Kang, S. Chen, S. Zhang, E.Y. Pun, K.W. Cheah, J. Li, Spin-enabled plasmonic metasurfaces for manipulating orbital angular momentum of light. Nano Lett. 13, 4148-4151 (2013)

19. G.Y. Lee, G. Yoon, S.Y. Lee, H. Yun, J. Cho, K. Lee, H. Kim, J. Rho, B. Lee, Complete amplitude and phase control of light using broadband holographic metasurfaces. Nanoscale (2018). http://dx.doi.org/10.1039/ C7NR07154J

20. G. Yoon, S. So, M. Kim, R. Ma, J. Rho, Electrically tunable metasurface perfect absorber for infrared frequencies, Nano Convergence 4, 36 (2017)

21. T. Badloe, J. Mun, J. Rho, Metasurfaces-based absorption and reflection control of light: perfect absorbers and reflectors. J. Nanomaterials 2017 $2361042(2017)$

22. S.J. Tan, X.M. Goh, Y.M. Wang, J.K.W. Yang, J. Teng, Engineering plasmonic colors in metal nanostructures. J. Mol. Eng. Mater. 2, 1440011 (2014)

23. T. Xu, H. Shi, Y. Wu, A.F. Kaplan, J.G. Ok, L.J. Guo, Structural colors: from plasmonic to carbon nanostructures. Small 7, 3128-3136 (2011)

24. W. Wang, D. Rosenmann, D. A. Czaplewski, X. Yang, and J. Gao, Realizing structural color generation with aluminum plasmonic $\mathrm{V}$-groove metasurfaces. Opt. Express 25, 20454-20465 (2017)

25. E. Stefan, P. Evgueni, N. Michel, R. Raymond, Enhanced light transmission by hole arrays. J. Opt. A. 4, S83 (2002)

26. W. Fan, S. Zhang, B. Minhas, K.J. Malloy, S.R.J. Brueck, Enhanced infrared transmission through subwavelength coaxial metallic arrays. Phys. Rev. Lett. 94, 033902 (2005)

27. H. Liu, P. Lalanne, Microscopic theory of the extraordinary optical transmission. Nature 452, 728-731 (2008)

28. C.S. Park, V.R. Shrestha, W. Yue, S. Gao, S.S. Lee, E.S. Kim, D.Y. Choi, Structural color filters enabled by a dielectric metasurface incorporating hydrogenated amorphous silicon nanodisks. Sci. Rep. 7, 2556 (2017)

29. A. Boltasseva, H.A. Atwater, Low-loss plasmonic metamaterials. Science 331, 290-291 (2011)

30. P.R. West, S. Ishii, G.V. Naik, N.K. Emani, V.M. Shalaev, A. Boltasseva, Searching for better plasmonic materials. Laser Photon. Rev. 4, 795-808 (2010)

31. Y. Kivshar, A. Miroshnichenko, Meta-optics with Mie resonances. Opt. Photonics News 28, 24-31 (2017)

32. R. Bartholomew, C. Williams, A. Khan, R. Bowman, T. Wilkinson, Plasmonic nanohole electrodes for active color tunable liquid crystal transmissive pixels. Opt. Lett. 42, 2810-2813 (2017)

33. J. Olson, A. Manjavacas, T. Basu, D. Huang, A.E. Schlather, B. Zheng, N.J. Halas, P. Nordlander, S. Link, High chromaticity aluminum plasmonic pixels for active liquid crystal displays. ACS Nano 10, 1108-1117 (2016)

34. G. Si, Y. Zhao, E.S.P. Leong, Y.J. Liu, Liquid-crystal-enabled active plasmonics: a review. Materials 7, 1296-1317 (2014)

35. Y.J. Liu, C.Y. Si, E.S.P. Leong, N. Xiang, A.J. Danner, J.H. Teng, Light-driven plasmonic color filters by overlaying photoresponsive liquid crystals on gold annular aperture arrays. Adv. Mater. 24, OP131-OP135 (2012)

36. Y. Shen, V. Rinnerbauer, I. Wang, V. Stelmakh, J.D. Joannopoulos, M. Soljačić, Structural colors from fano resonances. ACS Photonics 2, 27-32 (2015)

37. L. Zhu, J. Kapraun, J. Ferrara, C.J. Chang-Hasnain, Flexible photonic metastructures for tunable coloration. Optica 2, 255-258 (2015)

38. P. Gutruf, C. Zou, W. Withayachummnankul, M. Bhaskaran, S. Sriram, C. Fumeaux, Mechanically tunable dielectric resonator metasurfaces at visible frequencies. ACS Nano 10, 133-141 (2016)
39. J. Li, C.M. Shah, W. Withayachummnankul, B.S.Y. Ung, A. Mitchell, S. Sriram, M. Bhaskaran, S. Chang, D. Abbott, Mechanically tunable terahertz metamaterials. Appl. Phys. Lett. 102, 121101 (2013)

40. S.S. Mirshafieyan, J. Guo, Silicon colors: spectral selective perfect light absorption in single layer silicon films on aluminum surface and its thermal tunability. Opt. Express 22, 31545-31554 (2014)

41. V. Vashistha, G. Vaidya, P. Gruszecki, A. E. Serebryannikov, M. Krawczyk, Polarization tunable all-dielectric color filters based on cross-shaped Si nanoantennas. Sci. Rep. 7, 8092 (2017)

42. M.J. Uddin, T. Khaleque, R. Magnusson, Guided-mode resonant polarization-controlled tunable color filters. Opt. Express 22, 12307-12315 (2014)

43. S.S. Wang, R. Magnusson, Theory and applications of guided-mode resonance filters. Appl. Opt. 32, 2606-2613 (1993)

44. J. Braun, B. Gompf, G. Kobiela, M. Dressel, How holes can obscure the view: suppressed transmission through an ultrathin metal film by a subwavelength hole array. Phys. Rev. Lett. 103, 203901 (2009)

45. C. Genet, T.W. Ebbesen, Light in tiny holes. Nature 445, 39-46 (2007)

46. M. Jalali, Y. Yu, K. Xu, R.J.H. Ng, Z. Dong, L. Wang, S.S. Dinachali, M. Hong, J.K.W. Yang, Stacking of colors in exfoliable plasmonic superlattices. Nanoscale 8, 18228-18234 (2016)

47. E. Balaur, C. Sadatnajafi, S.S. Kou, J. Lin, B. Abbey, Continuously tunable, polarization controlled, colour palette produced from nanoscale plasmonic pixels. Sci. Rep. 6, 28062 (2016)

48. S. Yokogawa, S.P. Burgos, H.A. Atwater, Plasmonic color filters for CMOS image sensor applications. Nano Lett. 12, 4349-4354 (2012)

49. K. Walls, Q. Chen, S. Collins, D.R.S. Cumming, T.D. Drysdale, Automated design, fabrication, and characterization of color matching plasmonic filters. IEEE Photon. Technol. Lett. 24, 602-604 (2012)

50. Q. Chen, D. Das, D. Chitnis, K. Walls, T.D. Drysdale, S. Collins, D.R.S. Cumming, A CMOS image sensor integrated with plasmonic colour filters. Plasmonics 7, 695-699 (2012)

51. Q. Chen, D.R.S. Cumming, High transmission and low color cross-talk plasmonic color filters using triangular-lattice hole arrays in aluminum films. Opt. Express 18, 14056-14062 (2010)

52. Z. Li, A.W. Clark, J.M. Cooper, Dual color plasmonic pixels create a polarization controlled nano color palette. ACS Nano 10, 492-498 (2016)

53. B.R. Lu, C. Xu, J. Liao, J. Liu, Y. Chen, High-resolution plasmonic structural colors from nanohole arrays with bottom metal disks. Opt. Lett. 41, 1400-1403 (2016)

54. W. Yue, S. Gao, S.S. Lee, E.S. Kim, D.Y. Choi, Highly reflective subtractive color filters capitalizing on a silicon metasurface integrated with nanostructured aluminum mirrors. Laser Photon. Rev. 11, 1600285 (2017)

55. C.S. Park, V.R. Shrestha, S.S. Lee, D.Y. Choi, Trans-reflective color filters based on a phase compensated etalon enabling adjustable color saturation. Sci. Rep. 6, 25496 (2016)

56. M.A. Kats, F. Capasso, Optical absorbers based on strong interference in ultra-thin films. Laser Photon. Rev. 10, 735-749 (2016)

57. K.T. Lee, S. Seo, L.J. Guo, High-color-purity subtractive color filters with a wide viewing angle based on plasmonic perfect absorbers. Adv. Opt. Mater. 3, 347-352 (2015)

58. S.J.Tan, L. Zhang, D. Zhu, X.M. Goh, Y.M. Wang, K. Kumar, C.W. Qiu, J.K.W. Yang, Plasmonic color palettes for photorealistic printing with aluminum nanostructures. Nano Lett. 14, 4023-4029 (2014)

59. A.S. Roberts, A. Pors, O. Albrektsen, S.I. Bozhevolnyi, Subwavelength plasmonic color printing protected for ambient use. Nano Lett. 14, 783-787 (2014)

60. K.T. Lee, S. Seo, J.Y. Lee, L.J. Guo, Ultrathin metal-semiconductor-metal resonator for angle invariant visible band transmission filters. Appl. Phys. Lett. 104, 231112 (2014)

61. Y.T. Yoon, S.S. Lee, Transmission type color filter incorporating a silver film based etalon. Opt. Express 18, 5344-5349 (2010)

62. T. Xu, Y.K. Wu, X. Luo, L.J. Guo, Plasmonic nanoresonators for highresolution colour filtering and spectral imaging. Nat. Commun. 1, 1058 (2010)

63. F. Cheng, X. Yang, D. Rosenmann, L. Stan, D. Czaplewski, J. Gao, Enhanced structural color generation in aluminum metamaterials coated with a thin polymer layer. Opt. Express 23, 25329-25339 (2015)

64. F. Cheng, J. Gao, T. S. Luk, X. Yang, Structural color printing based on plasmonic metasurfaces of perfect light absorption. Sci. Rep. 5, 11045 (2015) 
65. R. Mudachathi, T. Tanaka, Up scalable full colour plasmonic pixels with controllable Hue, brightness and saturation. Sci. Rep. 7, 1199 (2017)

66. K. Walls, Q. Chen, J. Grant, S. Collins, D.R.S. Cumming, T.D. Drysdale, Narrowband multispectral filter set for visible band. Opt. Express 20 21917-21923 (2012)

67. B. Fang, C. Yang, W. Shen, X. Zhang, Y. Zhang, X. Liu, Highly efficient omnidirectional structural color tuning method based on dielectricmetal-dielectric structure. Appl. Opt. 56, C175-C180 (2017)

68. H. Wang, X. Wang, C. Yan, H. Zhao, J. Zhang, C. Santschi, O. J. F. Martin, full color generation using silver tandem nanodisks. ACS Nano 11, 4419-4427 (2017)

69. X.M. Goh, Y. Zheng, S.J. Tan, K. Kumar, C.W. Qiu, J.K.W. Yang Threedimensional plasmonic stereoscopic prints in full colour. Nat. Commun. 5, 6361 (2014)

70. W. Yue, S. Gao, S.S. Lee, E.S. Kim, D.Y. Choi, Subtractive color filters based on a silicon-aluminum hybrid-nanodisk metasurface enabling enhanced color purity. Sci. Rep. 6, 29756 (2016)

71. X.L. Hu, L.B. Sun, B. Zeng, L.S. Wang, Z.G. Yu, S.A. Bai, S.M. Yang, L.X. Zhao, Q. Li, M. Qiu, R.Z. Tai, H.J. Fecht, J.Z. Jiang, D.X. Zhang, Polarization-independent plasmonic subtractive color filtering in ultrathin Ag nanodisks with high transmission. Appl. Opt. 55, 148-152 (2016)

72. X.M. Goh, R.J.H. Ng, S. Wang, S.J. Tan, J.K.W. Yang, Comparative study of plasmonic colors from all-metal structures of posts and pits. ACS Photonics 3, 1000-1009 (2016)

73. V.R. Shrestha, S.S. Lee, E.S. Kim, D.Y. Choi, Aluminum plasmonics based highly transmissive polarization-independent subtractive color filters exploiting a nanopatch array. Nano Lett. 14, 6672-6678 (2014)

74. J. Olson, A. Manjavacas, L. Liu, W. Chang, B. Foerster, N.S. King, M.W. Knight, P. Nordlander, N.J. Halas, S. Link, Vivid, full-color aluminum plasmonic pixels. Proc. Natl. Acad. Sci. USA 111, 14348-14353 (2014)

75. G. Si, Y. Zhao, J. Lv, M. Lu, F. Wang, H. Liu, N. Xiang, T.J. Huang, A.J. Danner, J. Teng, Y.J. Liu, Reflective plasmonic color filters based on lithographically patterned silver nanorod arrays. Nanoscale 5 6243-6248 (2013)

76. T. Ellenbogen, K. Seo, K.B. Crozier, Chromatic plasmonic polarizers for active visible color filtering and polarimetry. Nano Lett. 12, 1026-1031 (2012)

77. K. Kumar, H. Duan, R.S. Hedge, S.C.W. Koh, J.N. Wei, J.K.W. Yang, Printing colour at the optical diffraction limit. Nat. Nanotechnol. 7, 557-561 (2012)

78. T.D. James, P. Mulvaney, A. Roberts, The plasmonic pixel: large area, wide gamut color reproduction using aluminum nanostructures. Nano Lett. 16, 3817-3823 (2016)

79. A.L. Fannin, B.R. Wenner, J.W. Allen, M.S. Allen, R. Magnusson, Properties of mixed metal-dielectric nanogratings for application in resonant absorption, sensing, and display. Opt. Eng. 56, 121905 (2017)

80. J. Wang, Q. Fan, S. Zhang, Z. Zhang, H. Zhang, Y. Liang, X. Cao, T. Xu Ultra-thin plasmonic color filters incorporating free-standing resonant membrane waveguides with high transmission efficiency. Appl. Phys. Lett. 110, 031110 (2017)

81. L. Duempelmann, D. Casari, A. Luu-Dinh, B. Gallinet, L. Novotny, Color rendering plasmonic aluminum substrates with angular symmetry breaking. ACS Nano 9, 12383-12391 (2015)

82. B. Zeng, Y. Gao, F.J. Bartoli, Ultrathin nanostructured metals for highly transmissive plasmonic subtractive color filters. Sci. Rep. 3, 02840 (2013)

83. Y.K.R. Wu, A.E. Hollowell, C. Zhang, L.J. Guo, Angle-insensitive structural colours based on metallic nanocavities and coloured pixels beyond the diffraction limit. Sci. Rep. 3, 01194 (2013)

84. M.J. Uddin, R. Magnusson, Efficient guided-mode-resonant tunable color filters. IEEE Photon. Technol. Lett. 24, 1552-1554 (2012)

85. A.F. Kaplan, T. Xu, L.J. Guo, High efficiency resonance-based spectrum filters with tunable transmission bandwidth fabricated using nanoimprint lithography. Appl. Phys. Lett. 99, 143111 (2011)

86. J.L. Skinner, A.A. Talin, D.A. Horsley, A MEMS light modulator based on diffractive nanohole gratings. Opt. Express 16, 3701-3711 (2008)

87. A.B. Christiansen, E. Højlund-Nielsen, J. Clausen, G.P. Caringal, N.A. Mortensen, A. Kristensen, Imprinted and injection-molded nano-structured optical surfaces, Proceedings, Nanostructured Thin Films VI, vol. 8818, 881803, (SPIE, San Diego, 2013)

88. V.R. Shrestha, S.S. Lee, E.S. Kim, D.Y. Choi, Polarization-tuned dynamic color filters incorporating a dielectric-loaded aluminum nanowire array. Sci. Rep. 5, 12450 (2015)
89. D.M. Nguyen, D. Lee, J. Rho, Control of light absorbance using plasmonic grating based perfect absorber at visible and near-infrared wavelengths, Sci. Rep. 7, 2611 (2017)

90. Q.J. Wu, H. Jia, X.L. Hu, L.B. Sun, L.S. Wang, S.M. Yang, R.Z. Tai, H.J. Fecht, L.Q. Wang, D.X. Zhang, Plasmonic reflection color filters with metallic random nanostructures. Nanotechnology 28, 085203 (2017)

91. L. Duempelmann, A. Luu-Dinh, B. Gallinet, L. Novotny, Four-fold color filter based on plasmonic phase retarder. ACS Photonics 3, 190-196 (2016)

92. G. Zheng, H. Mühlenbernd, M. Kenny, G. Li, T. Zentgraf, S. Zhang Metasurface holograms reaching 80\% efficiency. Nat. Nanotechnol. 10 308-312 (2015)

93. H. Lochbihler, Polarizing and angle-sensitive color filter in transmittance for security feature applications. Adv. Opt. Technol. 4, 71-77 (2015)

94. Y. Shen, J. Zhou, T. Liu, Y. Tao, R. Jiang, M. Liu, G. Xiao, J. Zhu, Z.K. Zhou, X. Wang, C. Jin, J. Wang, Plasmonic gold mushroom arrays with refractive index sensing figures of merit approaching the theoretical limit. Nat. Commun. 4, 3381 (2013)

95. M. Liu, X. Zhang, Nano-optics: plasmon-boosted magneto-optics. Nat. Photonics 7, 429-430 (2013)

96. A.C. Lowe (ed.), Liquid Crystal Displays: Addressing Schemes and ElectroOptical Effects (Wiley, New York, 2010)

97. M.A. van de Haar, J. van de Groep, B.J.M. Brenny, A. Polman, Controlling magnetic and electric dipole modes in hollow silicon nanocylinders. Opt. Express 24, 2047-2064 (2016)

98. Y.H. Fu, A.I. Kuznetsov, A.E. Miroshnichenko, Y.F. Yu, B. Luk'yanchuk, Directional visible light scattering by silicon nanoparticles. Nat. Commun. 4, 1527 (2013)

99. B.S. Luk'yanchuk, N.V. Voshchinnikov, R. Paniagua-Domínguez, A.I. Kuznetsov, Optimum forward light scattering by spherical and spheroidal dielectric nanoparticles with high refractive index. ACS Photonics $\mathbf{2}$ 993-999 (2015)

100. A.B. Evlyukhin, C. Reinhardt, B.N. Chichkov, Multipole light scattering by nonspherical nanoparticles in the discrete dipole approximation. Phys. Rev. B 84, 235429 (2011)

101. A.I. Kuznetsov, A.E. Miroshnichenko, M.L. Brongersma, Y.S. Kivshar, B. Luk'yanchuk, Optically resonant dielectric nanostructures. Science 354, aag2472 (2016)

102. G. Yoon, D. Lee, K.T. Nam, J. Rho, Pragmatic metasurface hologram at visible wavelength: the balance between diffraction efficiency and fabrication compatibility. ACS Photonics (2018). https://doi.org/10.1021/ acsphotonics.7b01044

103. Z. Li, I. Kim, L. Zhang, M.Q. Mehmood, M.S. Anwar, M. Saleem, D. Lee, K.T. Nam, S. Zhang, B. Luk'yanchuk, Y. Wang, G. Zheng, J. Rho, C. Qiu, Dielectric meta-holograms enabled with dual magnetic resonances in visible light, ACS Nano 11, 9382-9389 (2017)

104. J. Proust, F. Bedu, B. Gallas, I. Ozerov, N. Bonod, All-dielectric colored metasurfaces with silicon Mie resonators. ACS Nano 10, 7761-7767 (2016)

105. R. Regmi, J. Berthelot, P.M. Winkler, M. Mivelle, J. Proust, F. Bedu, I. Ozerov, T. Begou, J. Lumeau, H. Rigneault, M.F. García-Parajó, S. Bidault, J. Wenger, N. Bonod, All-dielectric silicon nanogap antennas to enhance the fluorescence of single molecules. Nano Lett. 16, 5143-5151 (2016)

106. M.K. Schmidt, R. Esteban, J.J. Sáenz, I. Suárez-Lacalle, S. Mackowski, J. Aizpurua, Dielectric antennas - a suitable platform for controlling magnetic dipolar emission. Opt. Express 20, 13636-13650 (2012)

107. M. Caldarola, P. Albella, E. Cortés, M. Rahmani, T. Roschuk, G. Grinblat, R.F. Oulton, A.V. Bragas, S.A. Maier, Non-plasmonic nanoantennas for surface enhanced spectroscopies with ultra-low heat conversion. Nat. Commun. 6, 8915 (2015)

108. B. Rolly, B. Bebey, S. Bidault, B. Stout, N. Bonod, Promoting magnetic dipolar transition in trivalent lanthanide ions with lossless Mie resonances. Phys. Rev. B 85, 245432 (2012)

109. X. Zambrana-Puyalto, N. Bonod, Purcell factor of spherical Mie resonators. Phys. Rev. B 91, 195422 (2015)

110. Z.J. Yang, R. Jiang, X. Zhuo, Y. Xie, J. Wang, H. Lin, Dielectric nanoresonators for light manipulation. Phys. Rep. 701, 1-50 (2017)

111. V. Vashistha, G. Vaidya, R.S. Hegde, A.E. Serebryannikov, N. Bonod, M. Krawczyk, All-dielectric metasurfaces based on cross-shaped resonators for color pixels with extended gamut. ACS Photonics 4, 1076-1082 (2017) 
112. V. Flauraud, M. Reyes, R. Paniagua-Domínguez, A.I. Kuznetsov, J. Brugger, Silicon nanostructures for bright field full color prints. ACS Photonics $\mathbf{4}$, 1913-1919 (2017)

113. T. Wood, M. Naffouti, J. Berthelot, T. David, J.B. Claude, L. Métayer, A. Delobbe, L. Favre, A. Ronda, I. Berbezier, N. Bonod, M. Abbarchi, Alldielectric color filters using SiGe-based Mie resonator arrays. ACS Photonics 4, 873-883 (2017)

114. H. Park, K. Seo, K.B. Crozier, Adding colors to polydimethylsiloxane by embedding vertical silicon nanowires. Appl. Phys. Lett. 101, 193107 (2012)

115. Y.T. Yoon, H.S. Lee, S.S. Lee, S.H. Kim, J.D. Park, K.D. Lee, Color filter incorporating a subwavelength patterned grating in poly silicon. Opt. Express 16, 2374-2380 (2008)

116. Y. Kanamori, M. Shimono, K. Hane, Fabrication of transmission color filters using silicon subwavelength gratings on quartz substrates. IEEE Photon. Technol. Lett. 18, 2126-2128 (2006)

117. B.H. Cheong, O.N. Prudnikov, E. Cho, H.S. Kim, J. Yu, Y.S. Cho, H.Y. Choi, S.T. Shin, High angular tolerant color filter using subwavelength grating. Appl. Phys. Lett. 94, 213104 (2009)

118. N. Dhindsa, S.S. Saini, Localized absorption in aluminum mask in visible spectrum due to longitudinal modes in vertical silicon nanowire arrays. J. Appl. Phys. 117, 224302 (2015)

119. S.C. Yang, K. Richter, W.J. Fischer, Multicolor generation using silicon nanodisk absorber. Appl. Phys. Lett. 106, 081112 (2015)

120. H. Park, K. B. Crozier, Multispectral imaging with vertical silicon nanowires. Sci. Rep. 3, 2460 (2013)

121. T. Bottein, T. Wood, T. David, J.B. Claude, L. Favre, I. Berbézier, A. Ronda, M. Abbarchi, D. Grosso, "Black" titania coatings composed of sol-gel imprinted mie resonators arrays. Adv. Funct. Mater. 27, 1604924 (2017)

122. P. Lalanne, S. Astilean, P. Chavel, E. Cambril, H. Launois, Design and fabrication of blazed binary diffractive elements with sampling periods smaller than the structural cutoff. J. Opt. Soc. Am. A 16, 1143-1156 (1999)

123. M. Khorasaninejad, W.T. Chen, R.C. Devlin, J. Oh, A.Y. Zhu, F. Capasso, Metalenses at visible wavelengths: diffraction-limited focusing and subwavelength resolution imaging. Science 352, 1190-1194 (2016)

124. C. Zou, P. Gutruf, W. Withayachumnankul, L. Zou, M. Bhaskaran, S. Sriram, C. Fumeaux, Nanoscale $\mathrm{TiO}_{2}$ dielectric resonator absorbers. Opt. Lett. 41, 3391-3394 (2016)

125. S. Sun, Z. Zhou, C. Zhang, Y. Gao, Z. Duan, S. Xiao, Q. Song, All-dielectric full-color printing with $\mathrm{TiO}_{2}$ metasurfaces. ACS Nano 11, 4445-4452 (2017)

126. P. Albella, R. Alcaraz de la Osa, F. Moreno, S.A. Maier, Electric and magnetic field enhancement with ultralow heat radiation dielectric nanoantennas: considerations for surface-enhanced spectroscopies. ACS Photonics 1, 524-529 (2014)

127. C.H. Park, Y.T. Yoon, V.R. Shrestha, C.S. Park, S.S. Lee, E.S. Kim, Electrically tunable color filter based on a polarization-tailored nano-photonic dichroic resonator featuring an asymmetric subwavelength grating. Opt. Express 21, 28783-28793 (2013)

128. D. Franklin, Y. Chen, A. Vazquez-Guardado, S. Modak, J. Boroumand, D. Xu, S. Wu, D. Chanda, Polarization-independent actively tunable colour generation on imprinted plasmonic surfaces. Nat. Commun. 6, 7337 (2015)

129. D. Franklin, R. Frank, S.T. Wu, D. Chanda, Actively addressed single pixel full-colour plasmonic display. Nat. Commun. 8, 15209 (2017)

130. G. Wang, X. Chen, S. Liu, C. Wong, S. Chu, Mechanical chameleon through dynamic real-time plasmonic tuning. ACS Nano 10, 1788-1794 (2016)

131. X. Duan, S. Kamin, N. Liu, Dynamic plasmonic colour display. Nat. Commun. 8, $14606(2017)$

132. Y. Chen, X. Duan, M. Matuschek, Y. Zhou, F. Neubrech, H. Duan, N. Liu, Dynamic color displays using stepwise cavity resonators. Nano Lett. 17, 5555-5560 (2017)

133. N. Raeis-Hosseini, J. Rho, Metasurfaces based on phase-change material as a reconfigurable platform for multifunctional devices, Materials $\mathbf{1 0}$ 1046 (2017)

134. A.E. Miroshnichenko, S. Flach, Y.S. Kivshar, Fano resonances in nanoscale structures. Rev. Mod. Phys. 82, 2257 (2010)
135. X. Zhu, W. Yan, U. Levy, N.A. Mortensen, A. Kristensen, Resonant laser printing of structural colors on high-index dielectric metasurfaces. Sci. Adv. 3, e1602487 (2017)

136. X. Zhu, C. Vannahme, E. Højlund-Nielsen, N.A. Mortensen, A. Kristensen, Plasmonic colour laser printing. Nat. Nanotechnol. 11, 325-329 (2015)

137. J. Xue, Z.K. Zhou, Z. Wei, R. Su, J. Lai, J. Li, C. Li, T. Zhang, X.H. Wang, Scalable, full-colour and controllable chromotropic plasmonic printing. Nat. Commun. 6, 9906 (2015)

138. Z. Li, S. Butun, K. Aydin, Large-area, lithography-free super absorbers and color filters at visible frequencies using ultrathin metallic films. ACS Photonics 2, 183-188 (2015)

139. Y.S. Do, J.H. Park, B.Y. Hwang, S. Lee, B. Ju, K.C. Choi, Plasmonic color filter and its fabrication for large-area applications. Adv. Opt. Mater. 1, 133-138 (2013)

140. J.M. Guay, A.C. Lesina, G. Côté, M. Charron, D. Poitras, L. Ramunno, P. Berini, A. Weck, Laser-induced plasmonic colours on metals. Nat. Commun. 8, 16095 (2017)

141. C. Kerse, H. Kalaycıoğlu, P. Elahi, B. Çetin, D.K. Kesim, Ö. Akçaalan, S. Yavaş, M.D. Aşık, B. Öktem, H. Hoogland, R. Holzwarth, F.Ö. Ilday, Ablationcooled material removal with ultrafast bursts of pulses. Nature $\mathbf{5 3 7}$ 84-88 (2016)

142. W. Hu, Y.C. Shin, G. King, Modeling of multi-burst mode pico-second laser ablation for improved material removal rate. Appl. Phys. A 98 , 407-415 (2010)

143. C. Hartmann, A. Gillner, Ü. Aydin, R. Noll, T. Fehr, C. Gehlen, R. Poprawe, Investigation on laser micro ablation of metals using ns-multi-pulses. J. Phys.: Conf. Ser. 59, 440 (2007)

144. H. Im, K.C. Bantz, S.H. Lee, T.W. Johnson, C.L. Haynes, S. Oh, Self-assembled plasmonic nanoring cavity arrays for SERS and LSPR biosensing. Adv. Mater. 25, 2678-2685 (2013)

145. X. Ye, L. Qi, Two-dimensionally patterned nanostructures based on monolayer colloidal crystals: controllable fabrication, assembly, and applications. Nano Today 6, 608-631 (2011)

146. M. Bardosova, M.E. Pemble, I.M. Povey, R.H. Tredgold, The LangmuirBlodgett approach to making colloidal photonic crystals from silica spheres. Adv. Mater 22, 3104-3124 (2010)

147. S.H. Lee, K.C. Bantz, N.C. Lindquist, S. Oh, C.L. Haynes, Self-assembled plasmonic nanohole arrays. Langmuir 25, 13685-13693 (2009)

148. L. Wang, R.J.H. Ng, S.S. Dinachali, M. Jalali, Y. Yu, J.K.W. Yang, large area plasmonic color palettes with expanded gamut using colloidal selfassembly. ACS Photonics 3, 627-633 (2016)

149. H. Galinski, G. Favradu, H. Dong, J.S.T. Gongora, G. Favaro, M. Döbeli, R. Spolenak, A. Fratalocchi, F. Capasso, Scalable, ultra-resistant structural colors based on network metamaterials. Light Sci. Appl. 6, e16233 (2017)

\section{Submit your manuscript to a SpringerOpen ${ }^{\circ}$ journal and benefit from:}

- Convenient online submission

- Rigorous peer review

- Open access: articles freely available online

- High visibility within the field

- Retaining the copyright to your article

Submit your next manuscript at springeropen.com 